\title{
Folgen von Leistungsbewertungen für ProfessorInnen: Drei Fallbeispiele
}

In diesem Kapitel wollen wir anhand ausgewählter Fälle in die Betrachtung der positiven, negativen oder ambivalenten Folgen einsteigen, die sich für ProfessorInnen mit der neuen Governance und insbesondere universitären Leistungsbewertungen verbinden. Anhand von drei ausführlichen Fallbeispielen soll das Verhältnis zwischen den professoralen Ansprüchen an ,gute` Lehre und Forschung auf der einen und den organisationalen Bewertungsmaßstäben und Leistungskriterien auf der anderen Seite nachgezeichnet werden. Ausgehend von der grundsätzlichen Einschätzung der universitären Wandlungsprozesse, nach der in den Interviews jeweils zu Beginn gefragt wurde, werden wir darstellen, wie ProfessorInnen die geänderten universitären Leistungsbewertungen in den beiden Kernaufgaben der Lehre und Forschung in Abhängigkeit von eigenen Qualitätsmaßstäben für sich deuten und verarbeiten.

Dazu haben wir zunächst zwei kontrastive Fallbeispiele aus Disziplinen gewählt, die sich hinsichtlich ihrer Wissenschaftskultur und ihrer Nähe bzw. Distanz zu quantifizierenden Kriterien der Leistungsbewertung - wie etwa Drittmittelvolumina - stark unterscheiden. ${ }^{1}$ Ein dritter, in der Mitte zwischen diesen beiden angesiedelter Fall verdeutlicht die den gegenwärtigen Reformprozess kennzeichnenden Ambivalenzen und Widersprüche in besonderer Weise. Bei der Fallauswahl beziehen wir zudem unterschiedliche Karrierephasen mit ein, um der naheliegenden Vermutung Rechnung zu tragen, dass die subjektive Aneignung universitärer Leistungsbewertungen nicht allein von - über Disziplin und

\footnotetext{
${ }^{1}$ Wir sprechen der Disziplin hier eine prägende, aber keine determinierende Kraft zu, da sich, wie wir in Kap. 3 gezeigt haben, auch innerhalb einer Disziplin differierende Deutungen, guter' Forschung und Lehre finden lassen.
} 
berufliche Sozialisation vermittelten - Qualitätsmaßstäben abhängt, sondern auch von dem jeweils wahrgenommenen Druck, sich mit Blick auf die eigene inneruniversitäre Position und weitergehende Karriereschritte noch bewähren zu müssen. Angesichts der forcierten Bedeutung bestimmter, für den universitären Wettbewerb wichtiger, Forschungsleistungen ist in diesem Kontext zudem die Frage interessant, ob dadurch die Balance zwischen Lehre und Forschung verändert, etwa der zeitliche Aufwand für die Lehrtätigkeit reduziert wird.

\subsection{Herr Timme: Leistungsbewertungen als Bestätigung der beruflichen Identität? (TECH6) ${ }^{2}$}

Beginnen wollen wir mit dem Fallbeispiel von Herrn Timme, der ein W3-Professor für Nachrichtentechnik und bereits seit längerem an seiner jetzigen Universität tätig ist. Anders als neu berufene ProfessorInnen sieht er sich einem geringen Druck ausgesetzt, sich an seiner Universität noch bewähren - oder, wie Herr Timme es nennt, erst „freischwimmen“ - zu müssen. Hinzu kommt, dass quantifizierende Bewertungskriterien wie Drittmittel oder Promovierendenzahlen unproblematisch für ihn sind. Solche Kriterien repräsentierten zwar „nie das ganze Bild“. Für einen Vergleich der Forschungs- und Lehrleistungen elektrotechnischer Fakultäten stellten sie aber durchaus ,eine sinnvolle Metrik“ dar.

Vor seiner Berufung verbrachte Herr Timme mehrere Jahre bei einem größeren Industrieunternehmen. Die Wettbewerbs- und Kennzahlenlogik, die im Sinne des „New Public Management“ (NPM) auch auf Universitäten übertragen wurde, ist für ihn insofern nichts grundsätzlich Neues. Es ließe sich daher vermuten, dass er sich die universitären Leistungsbewertungen und -anreize durchaus in einer strategischen Weise aneignet.

Deutung universitärer Wandlungsprozesse: Ökonomischer ,Pay Off‘ und größere Autonomie

Diese Vermutung scheint sich zu Beginn des Interviews zu bestätigen. Denn Herr Timme antwortet auf die Einstiegsfrage nach seiner subjektiven Wahrnehmung universitärer Wandlungsprozesse mit folgender Einschätzung:

\footnotetext{
${ }^{2}$ Anders als im vorherigen und in den beiden nachfolgenden Kapiteln wird in diesem Kapitel aufgrund der Fokussierung auf drei Fälle auf weitere Interviewkennungen im Fließtext verzichtet. Stattdessen wird in den entsprechenden Unterkapiteln die Interviewkennung des dort ausführlich dargelegten Fallbeispiels jeweils eingangs einmalig genannt.
} 
„Ja, in der Tat, es gibt sehr viel Wandel. Einige versuche ich auszublenden und andere begrüße ich mit Nachdruck. Also das ist wirklich eine ganz große Spannbreite. Ich fange mal bei den positiven Aspekten an. Also ich selber bin W-besoldet und habe mir das nicht ausgesucht. Das war einfach irgendwann der Umbruch, wo es die C-Besoldung nicht mehr gab. Erst dachte ich, so noch in der Bewerbungsphase und wenn man so zum Vorsingen kam, das sei nachteilig, weil das Grundgehalt ist ja doch auch recht niedrig. Aber in den Ingenieurwissenschaften kommen doch die Berufungen weitestgehend aus der Industrie. Ich selber war auch lange Jahre bei [Name Unternehmen]. Und dann kann man halt schon, hat man schon eine gewisse Verhandlungsposition, indem man halt mal so seine letzten Gehälter auflegt. Und das habe ich auch ganz konkret getan. Und kurz bevor es zum Streit [...] kam, haben wir dann den Handschlag gemacht. Also das ist insofern eine, würde ich sagen, für die Ingenieurwissenschaften würde ich das als unkritisch beurteilen. Und ich gehe noch weiter. Es ist nicht nur unkritisch, sondern es ist ganz toll.“

Die ,positiven Aspekte“ des Wandels bestimmt Herr Timme hier über ökonomische Vorteile, die sich für ihn aus der W-Besoldung ergeben. Er profitierte in den Berufungsverhandlungen letztlich von den größeren Handlungsspielräumen des Präsidiums, auf seine „letzten Gehälter“ in der Wirtschaft eingehen zu können.

Das Thema materieller Vorteile spielt auch im Weiteren eine Rolle. Denn Herr Timme fährt unmittelbar damit fort, dass er seither zudem die Möglichkeit habe, über Industrieprojekte individualisierte „Forschungszulagen einzuwerben“. 3 Gerade diese führten dazu, sich über „Finanzspritzen“ freuen zu können, die ihn ,weit über eine C4-Besoldung katapultier[t]en“ und ein ,echter Leistungsanreiz" seien. Diese Deutung der Forschungszulagen ist bemerkenswert, wenn man sich die in Kap. 2 referierte vehemente Kritik an der universitären Anreizlogik in Erinnerung ruft, die von ProfessorInnen in der Zeitschrift Forschung und Lehre (F\&L) geäußert wurde. Herr Timme scheint demgegenüber einem unternehmerischen Ethos zu folgen und hierin keinen Widerspruch zum Status und Selbstverständnis eines Professors zu sehen.

Interessant ist allerdings, dass er als wichtigen Vorteil solcher Industrieprojekte auch die größere Autonomie gegenüber seiner Universität betrachtet. Trotz der mit den Kooperationspartnern vertraglich festgelegten Leistungen habe er selbst es letztlich ,zu hundert Prozent im Griff“, und es ,,befinde nicht irgendein Gremium darüber", wie seine „Performance war":

\footnotetext{
${ }^{3}$ ProfessorInnen, die nach Bundesbesoldungsordnung W berufen sind, können für die Dauer einer Projektfinanzierung im Bereich der Auftragsforschung eine nicht ruhegehaltsfähige Leistungszulage beanspruchen (Bundesbesoldungsgesetz: $§ 35$ ).
} 
„Und wenn man die Drittmittel einspielt und entsprechend dann auch eine Arbeitsgruppe hat, die jetzt nicht nur aus den Landesstellen besteht ((lacht)), dann wird man formal ziemlich in Ruhe gelassen. Das kann man schon sagen.“

Weitgehend offen ist damit jedoch noch, wie diese Form der Abgrenzung bei ihm motiviert ist - ob Herr Timme gegenüber einer organisationalen Kontrolle und Bewertung vor allem seinen professoralen Status oder auch inhaltliche Ansprüche an seine Forschung behauptet.

\section{Lehrqualität über intrinsische Motivation, Authentizität und Austausch mit den Studierenden}

In Bezug auf die universitären Leistungsbewertungen im Bereich der Lehre zeigen sich bei Herrn Timme zunächst keine größeren Vorbehalte. So bewertet er es als positiv, dass die Lehre mittlerweile mehr „Wertschätzung“ erfahre und an seiner Universität neben Evaluationen auch MentorInnengruppen eingeführt worden seien, in denen Studierende Schwierigkeiten mit Lehrenden thematisieren könnten. Durch die Kombination von formalisierter Bewertung und Möglichkeiten des informellen Austauschs habe man eine „ganz gute Handreichung“, um Probleme in der Lehre beheben zu können. Dadurch trage man auch dazu bei, die Universität zu einem attraktiven Studienort werden zu lassen und eine größere „Auslastung“ der Studiengänge zu erreichen.

Selbst positioniert sich Herr Timme als Angehöriger einer Disziplin, in der eine grundsätzliche Motivation für die Lehre schon durch die Abhängigkeit der Forschung von möglichst guten NachwuchswissenschaftlerInnen mehrheitlich gegeben sei:

„Wir wollen wirklich tolle Forschung betreiben. Wie kommen wir dahin? Wir brauchen gute Doktoranden. Wie kommen wir dahin? Wir müssen die Leute für uns ausbilden, für unsere Belange. [...] Also das kann man niemandem unterstellen, dass er da nachlässig ist oder eine schlechte Lehre macht."

Er legt hier ein „originäre[s] Eigeninteresse“ zugrunde, das sich bereits in den in Kap. 3 skizzierten Selbstdeutungen von Natur- und IngenieurwissenschaftlerInnen widerspiegelte.

Wenn er auf seine Veranstaltungen zu sprechen kommt, äußert sich bei Herrn Timme jedoch auch eine über die konkrete Lehrtätigkeit vermittelte Sinnstiftung. So schildert er mit Blick auf seine Vorlesungen:

„Ich starte, und dann der nächste Augenblick, wo ich mich bewusst wahrnehme, ist eineinhalb Stunden später. Also ich bin so absolut im Flow, was ein schönes 
Gefühl ist. Also das ist für mich, mehr so ein Dopamin-Ding ((lacht)). Also ich brauche Spaß. In zweiter Linie sollen die Studenten auch was mitnehmen, und das Schöne ist, dass das ja neurowissenschaftlich offenbar gekoppelt ist ((lacht)) - wenn der Lehrende Spaß hat, dass auch die Zuhörer ein bisschen Spaß haben und wach bleiben und Dinge mitkriegen.“

Entscheidend für eine erfolgreiche Wissensvermittlung ist für ihn seine eigene Begeisterung für die Materie. Durch diese gerate er bei seinen Vorlesungen in einen „Flow“, der sich in positiver Weise auf die Studierenden und deren Aufnahmebereitschaft bzw. -fähigkeit übertrage. Wenn diese ihn ,,an der Tafel tanzen“ sähen, leisteten sie mitunter mehr als das Geforderte. Ohne dass sie es müssten, setzten sich einige von ihnen etwa ,zu Hause hin und programmier[t]en das alles nach“. Im Gegenzug profitiere auch er, wenn sich die Studierenden in den Vorlesungen aktiver beteiligten und diese dadurch einen dialogischeren Charakter bekämen.

Für Herrn Timme hängt eine solche Wechselwirkung vor allem davon ab, die eigene intrinsische Motivation ,ein Stück rüber[zu]bringen“, während der konkrete didaktische „Weg“ eher „,sekundär“ sei. Insofern sieht er Weiterbildungsangebote, die im Sinne einer Verbesserung der Lehre qua „Hochglanzflyern“ an die ProfessorInnen herangetragen würden, eher kritisch. Das sei zwar „alles schön“, und er schätze das große Engagement des Prodekans für Studium und Lehre sehr. Wichtig sei aber, dass „das, was man einsetz[e]“, auch zur eigenen Person passe, man als ProfessorIn also weiterhin einem authentischen Lehrstil folge:

\begin{abstract}
„Sie gehen ins Geschäft, und Sie kaufen sich Kleidung, wo Sie denken, das unterstreicht Ihren Typ. Und da reicht nicht, da langt nicht irgendein bunter Blumenstrauß an, sage ich mal, Lehrmöglichkeiten. Und wenn mir die irgendjemand vorschreiben würde, dann würde ich ihm den Vogel zeigen. Ich ziehe die Kleidung an, die mir passt, und die ist recht konventionell eigentlich. Und das kommt auch dann genau so an, und ich bin überzeugt, da muss jeder seinen Weg finden.“
\end{abstract}

Herr Timme behauptet an dieser Stelle seine eigene, aber auch die Autonomie seiner KollegInnen gegenüber Versuchen, die Lehre gemäß neuen Methoden stärker zu standardisieren. Didaktischer Standardisierung wird die persönliche Erfahrung und Authentizität entgegengehalten. Zur Bekräftigung führt er das Beispiel älterer ProfessorInnen seiner Fakultät an, die zwar ,von der Methodik sehr konventionell“, bei den Studierenden jedoch „superbeliebt“ seien, da sie „für ihr Fach brennen" würden. Er wertet im Gegenzug jene KollegInnen nicht ab, die solch „schicke“ Methoden wie etwa „Votings“ via „Smartphone“ in ihre Veranstaltungen integrierten. Herr Timme distanziert sich aber von der Vorstellung, 
dass neue Formen der Didaktisierung zwangsläufig zu einer Verbesserung der Lehrqualität führten. Dagegen sprächen auch fachliche Gründe, die er wie folgt ausführt:

„Bei uns geht es viel um mathematische Dinge, da kann man nicht voten. Also da müssen die Leute mit Formeln irgendwie umgehen lernen. Also dass sie sich eher das Handgelenk wundschreiben, was ich auch wichtig finde. Ich sage immer: ,Laptops weg, Elektronik weg, ihr sollt schreiben." Also, wenn sich da motorisch etwas bei euch bewegt, dann speichert sich das ab. “”

Dass er selbst, wie er an anderer Stelle resümiert, „Fan der Tafel“ bleibe und auf „schicke Formen multimedialer Lehre" verzichte, ist bei Herrn Timme somit in zweifacher Weise motiviert. Zum einen sieht er den Lernerfolg bei bestimmten Inhalten nur durch klassische Methoden gewährleistet, zum anderen folgt er damit dem Anspruch, seinem eigenen Stil treu zu bleiben.

\section{Studierende als legitime Bewertungsinstanz}

Wichtig ist, dass Herr Timme sich jedoch nicht von einer Bewertung seiner Veranstaltungen durch die Studierenden abgrenzt. Für ihn sind die Lehrevaluationen vielmehr Ausdruck einer positiven Entwicklung des Verhältnisses zwischen Lehrenden und Lernenden. Dieses sei mittlerweile auch jenseits der Lehrveranstaltungen durch einen Austausch geprägt, der während seines eigenen Studiums noch weitgehend gefehlt habe. So äußert Herr Timme mit Blick auf die damalige Situation:

„Es gab schlichtweg keinen Kontakt. Ich habe seinerzeit die beste Klausur in der Mechanik geschrieben, die höchste Punktzahl, da hat bei mir ein Sternchen im Aushang gestanden, da habe ich dann ein Gespräch beim Professor bekommen. [...] Aber das war mein einziger Professoren-Kontakt im Grundstudium.“

Seither habe eine Enthierarchisierung stattgefunden, durch die sich beide Seiten - ProfessorInnen und Studierende - stärker ,auf Augenhöhe“ begegnen könnten. Herr Timme schreibt hierbei auch seiner Universität eine positive Rolle zu, die diese Entwicklung etwa durch die Einführung der bereits genannten MentorInnengruppen weiter fördere. Studierende könnten dort Themen besprechen, ,die ihnen auf dem Herzen liegen“, und im Gegenzug von ProfessorInnen ,sehr proaktiv“ in Bezug auf Hilfestellungen, aber auch Karriereoptionen am Fachbereich „,beraten“ werden.

Mit den Lehrevaluationen werde eine weitere Möglichkeit für Studierende geschaffen, Wünsche und Kritik - wenn auch vermittelt über ein standardisiertes 
Instrument - zu äußern. Dass sie die fachliche Kompetenz der ProfessorInnen nur bedingt einschätzen könnten, ist für Herrn Timme kein Argument gegen die Evaluationen:

„Ja, naturgemäß können die das nur teils beurteilen. Wenn Studenten schreiben, der Lehrende ist kompetent, dann können sie eigentlich nur sagen, er wirkt kompetent. $\mathrm{Ob}$ er kompetent ist, haben die keine Ahnung. Woher sollen sie das wissen? Also das ist natürlich nur so ein Schein. Aber auch der spielt, wenn wir uns als Dienstleister den Studierenden gegenüber verstehen wollen, schon eine Rolle. [...] Ob ich kompetent wirke oder ob ich motiviere oder ob der Lehrende häufig wechselt, das ist auf jeden Fall ein Qualitätsmerkmal einer Lehrveranstaltung, dass der Professor nie da ist, immer auf Dienstreise und einen Assistenten schickt und so, ne? Das ist schon eine Metrik, die die Studis sehr gut bewerten können. ${ }^{\text {}}$

Herr Timme geht somit eher von legitimen Ansprüchen der Studierenden an die Lehre aus, denen man durch die Evaluationen eine gewisse Geltung verschaffe. Für ihn selbst sei dieses Instrument in zweifacher Hinsicht ,hilfreich und motivierend“. Zum einen, weil er teilweise ,gute Hinweise“ bekäme, die er zukünftig in seinen Lehrveranstaltungen berücksichtigen könne. Zum anderen aber auch, weil seine Ergebnisse zumeist positiv ausfielen. An seiner Universität gäbe es alternativ auch die Option, mit ausgewählten Studierenden ein „Nachgespräch zu führen“. Er selbst sei jedoch ein „Freund von Zahlen“ und verzichte daher auf diese Möglichkeit:

„Wer gute Noten hat, wünscht sich kein Textzeugnis, sondern wünscht sich ein Notenzeugnis, verstehen Sie? ((lacht)) Und deswegen bevorzuge ich das Notenzeugnis. Das hat sich bewährt und damit kann ich meine Statistiken weiterschreiben.“

Insgesamt wird deutlich, dass sich Herr Timme die Leistungsbewertungen und das organisationale Ziel der Qualitätssicherung und -verbesserung in der Lehre positiv zu eigen machen kann. Angebote der didaktischen Weiterbildung sieht er zwar etwas kritisch, da er eher von der Deutung ausgeht, dass wissenschaftliche Persönlichkeiten schon durch ihre Begeisterung für die Sache - ihren „Tanz“ vor den

\footnotetext{
${ }^{4}$ Die Selbstbeschreibung als „Dienstleister“ ist bemerkenswert, stellt sie doch einen Gegensatz zu den sowohl im zuvor nachgezeichneten Diskurs (Kap. 2) als auch in den eigenen Interviews beschriebenen Selbstdeutungen dar. Ähnliche Beobachtungen finden sich auch bei Karin Lohr et al. (2013a, S. 157-162), die feststellten, dass Lehrende entsprechende Zuschreibungen explizit zurückwiesen, da diese im Widerspruch zu ihrem professionellen Selbstverständnis stünden.
} 
Studierenden - zu , guter', motivierender Lehre befähigt seien. Wichtiger scheint aber, dass Leistungsbewertungen wie Evaluationen seinen Vorstellungen eines zum Besseren gewandelten Verhältnisses zwischen Lehrenden und Lernenden weitgehend entsprechen: Anstatt den Status von ProfessorInnen gegenüber einer studentischen Bewertung in Stellung zu bringen, geht Herr Timme stärker von der professoralen Pflicht aus, den Lehrauftrag in verantwortungsvoller Weise zu erfüllen und Wünsche und Bedarfe der Studierenden zu berücksichtigen.

Zugleich verbindet er die Evaluationen mit einer strategischen Funktion. Denn gute Bewertungsergebnisse bedeuten für Herrn Timme und seine Fakultät auch die Chance, für Studierende attraktiv zu sein und darüber eine möglichst hohe Auslastung der Studierendenzahlen zu erreichen:

„Je mehr, desto besser. Also wir würden nie jemanden ablehnen. Das ist ganz klar, das ist auch eine Selektion, wir wollen eben auch wirklich sehr gute Kandidaten dann haben für Promotionen als Mitarbeiter. Und das geht nur, wenn Sie auch eine gewisse Masse haben."

,Gute ' Lehre ist für Herrn Timme damit zwar kein reines Mittel zum Zweck , guter Forschung. In seinen positiven Deutungen der Evaluationen spiegeln sich neben identitätsbezogenen Selbstansprüchen jedoch auch solche interessengeleiteten Motive wider.

\section{Forschung im eigenen Team anstelle ineffizienter Profilbildung}

Kritischer zeigt sich Herr Timme hingegen, wenn es um die organisationalen Ziele und Bewertungsmaßstäbe im Bereich der Forschung geht. Dies mag zunächst überraschen, da er Drittmittel, wie zu Beginn ausgeführt, als Leistungskriterium akzeptiert und zudem bei deren Einwerbung gut dasteht. Im Folgenden wird jedoch deutlich, dass sich seine Vorbehalte vor allem auf Profilbildung und groß angelegte Forschungsformate beziehen, an denen Universitäten im Sinne ihrer Sichtbarkeit und Wettbewerbsposition ein besonderes Interesse haben. Herr Timme lehnt einen „Findungsprozess“ zugunsten eines klareren Forschungsprofils zwar nicht grundsätzlich ab. Die damit verbundenen langwierigen Diskussionen und Versuche der erfolgreichen Institutionalisierung von Forschungsschwerpunkten sind für ihn aber mehr als kritikwürdig:

„Ich habe keine Lust, an diesen Prozessen mehr teilzunehmen in besonderer Weise. Ich habe mich viel engagiert, ich will jetzt auch keine Begriffe oder Namen hier nennen, das tut nichts zur Sache, aber Sie können sich, glaube ich, vorstellen, was das so für, da werden dann Zentren gebildet, man wird Mitglied in einem dieser Zentren oder überlegt sich, dort Mitglied zu werden oder engagiert sich dort auch 
vielleicht durch irgendwelche Ämter oder so. Ich meine, es gibt Meetings mit Kollegen, was könnten wir mal machen, um irgendwie mal einen Projektantrag zu schreiben? Ich [...] habe Stunden, Tage, Wochen, wahrscheinlich in Summe Monate in solchen Meetings gesessen und nicht einen einzigen Euro eingeworben über diese Wege. Im Gegenteil, ich habe sogar Beiträge für Zentren bezahlt aus meinen Restmitteln. Das bringt's alles nicht. Wenn ich aber meinen eigenen Impulsen gefolgt bin und auf Unternehmen zugegangen bin oder Anträge bei der DFG geschrieben habe, die bis auf einen alle zuerkannt worden sind, dann hat es was gebracht, dann hat es uns weitergebracht."

Herr Timme positioniert sich hier als Professor, der den geringen Mehrwert solcher „Prozesse“ aufgrund konkreter Erfahrungen beurteilen kann und sich insofern nicht per se gegenüber Neuem verweigert. Er habe schließlich viel Zeit in letztlich ergebnislosen „Meetings“ verbracht. Erfolgreich und effizient sei er hingegen bei seinen individuellen Bemühungen um Drittmittel gewesen. Da er „das Maß [s]eines Engagements“ in der universitären Verbundforschung selbst bestimmen könne, ziehe er für sich entsprechende Konsequenzen:

„Also das kann ich entscheiden, und dann guckt man, da gibt es so einen Zähler und einen Nenner in der Rechnung, und in dem Zähler ist so die Hoffnung auf mögliche Drittmittel, die man vielleicht gemeinschaftlich besser einwerben kann als alleine. Und im Nenner ist halt der zeitliche Aufwand. Das ist eine kühle Kalkulation. Wo sich mittlerweile der Nenner für mich zunehmend größer darstellt, weil im Zähler nicht viel steht.“

Allerdings setzt sich Herr Timme nicht nur in dieser selbstunternehmerisch anmutenden Weise mit der universitären Profilbildung auseinander. Denn er wirft zugleich die Frage auf, wo hierbei ,der Wert in Richtung der wirklich guten Forschung entsteh[e]“. Wie folgende Äußerung zeigt, behauptet er mit seiner Verweigerung gerade auch inhaltliche Qualitätsmaßstäbe:

„Ja, es entsteht Blindleistung, indem man sich profilieren muss gegenüber anderen Universitäten. Wozu? Wollen wir nicht einfach forschen und uns durch das, was an Forschungsleistung entsteht, profilieren? An Ehrungen durch Peers international orientieren? Das ist das, wie man Forschungsleistung am besten eigentlich bemisst, wie ich finde. Internationale Ehrungen oder Best Paper Awards oder irgendwie so etwas, danach sollten wir uns ausstrecken und danach wird eine Universität oder auch nur ein Fachbereich oder von mir aus ein Professor zum Leuchtturm und sichtbar werden und Studenten anwerben, motivieren, dort vielleicht zu studieren.“

Herr Timme stellt dem organisationalen Ziel der Profilbildung hier die Bewährung und Reputation innerhalb der Scientific Community gegenüber. Aus 
seiner Sicht werde in letzterer über die eigentliche Qualität der Forschung entschieden, wovon dann - im Erfolgsfall - auch die jeweilige Universität oder der Fachbereich profitieren könne. So kenne er Beispiele von ProfessorInnen, die „an irgendeiner B-Universität in der Bretagne“ tätig waren und dort zu wichtigen Ergebnissen gekommen seien, die sie zu den „Stars schlechthin“ hätten werden lassen. Herr Timme grenzt sich nicht grundsätzlich von den Interessen seiner Universität oder der Idee des universitären Wettbewerbs ab. Er verteidigt hier jedoch den Anspruch, die Forschung in erster Linie an wissenschaftlich fundierten Bewertungs- und Vergleichsmaßstäben zu orientieren.

Er konkretisiert seine Vorstellungen ,guter', sinnstiftender Forschung im Weiteren. Bei der Frage nach besonders positiven Erfahrungen kommt er nicht zuletzt auf das enge Verhältnis zu seinen Mitarbeitenden zu sprechen. Dass er als Professor und Projektleiter mittlerweile, wie auch andere FachkollegInnen, weniger in die alltägliche Programmierarbeit involviert ist, deutet Herr Timme nicht im Sinne eines Mangels. Denn durch den direkten Austausch mit seinem Team über Probleme oder Zwischenergebnisse bleibe er weiterhin mit dem Forschungsprozess verbunden:

\begin{abstract}
„Und die schönste Situation ist, dass ohne Termin oder irgendwas ein Mitarbeiter von mir mein Büro stürmt und sagt, er hat einen Bug gefunden ((lacht)). Das bedeutet, wir sind weitergekommen, ja? Irgendwas wird besser, oder bringt Kurven rein, und wir machen eine Fünf-Minuten-Spontandiskussion. Das ist eigentlich, wie ich mir meinen Beruf wünsche. Das ist sehr individuell. [...] Es gibt eben den ganzen Bereich zwischen Staatssekretär, Kanzler und Präsident. Wahrscheinlich bin ich mehr der Staatssekretär. Ich möchte diese Nähe zu meinen Assistenten, weil ich glaube, das ist ja das ganz besondere des Dr.-Ing. 's, den wir in Deutschland haben, dass man so ein Meister-Lehrling-Verhältnis hat und dieses Meister-Lehrling-Verhältnis funktioniert durch face time, funktioniert durch Kontakt, durch Gespräche.“
\end{abstract}

Über die „Nähe“ zu seinem Team behält Herr Timme einen stärkeren inneren Bezug zu dem jeweiligen Forschungsprojekt als ProfessorInnen, die eine vor allem manageriale Rolle einnehmen. Die kleinen Teilerfolge von Mitarbeitenden werden von ihm im Sinne eines gemeinsamen Fortschritts gedeutet, der auch ihn unmittelbar freue. Darüber hinaus kann Herr Timme durch die erwähnte „Nähe“ aktiv Anteil an der wissenschaftlichen Sozialisation seiner NachwuchswissenschaftlerInnen nehmen, was er durch den Vergleich mit einem „Meister-Lehrling-Verhältnis“ zusätzlich unterstreicht. In „Spontandiskussionen“ und durch regelmäßigen Austausch könnten die Mitarbeitenden sowohl von seiner Begeisterung für die Sache als auch von seinem Wissen profitieren. 
Dieses Rollenverständnis wird noch deutlicher, wenn Herr Timme sein Engagement bei der Arbeit an Publikationen beschreibt. So hat er zum einen den Anspruch, selbst „Ideen reinzureichen“. Zum anderen möchte er seinem Team in einem gemeinsamen Prozess des „Korrigierens, des Paper Herzeigens und Versionen Durcheditierens“ die Relevanz und Praxis des Publizierens in actu vermitteln:

\begin{abstract}
„Also ich predige immer wieder, wer nicht schreibt, der forscht nicht. Die denken immer, sie lesen Papers und programmieren was und das ist Forschung. Das stimmt nicht. Wenn die nicht geschrieben haben, dann werden ihnen wesentliche Lücken nicht klar. Weil ein wissenschaftliches Werk, und wenn es nur ein Tagungspaper ist, ist immer eine Story, ist eine Geschichte. Und die muss einen roten Faden haben und die hat einen Anfang und ein Ende, die hat einen Spannungsbogen, und diese Geschichte muss man erzählen können und entsprechend muss man die Simulationen aufbauen."
\end{abstract}

Wie wir in Kap. 3 bereits an Beispielen illustriert haben, spielt in den Ingenieurwissenschaften der Anspruch eines Anwendungsbezugs der Forschung häufiger eine wichtige Rolle. Dieser Anspruch spiegelt sich ebenfalls in den Deutungen ,guter" Forschung von Herrn Timme wider. Er äußert zwar mehrfach, dass die erfolgreiche Einreichung eines „Journal Paper immer ein sehr schöner Moment“ ein „echter Glücksmoment“ - sei. Gleichwohl orientiert er sich bei seiner Forschung nicht allein daran, sich vor dem kritischen Urteil der wissenschaftlichen Peers zu bewähren. „Sehr zufrieden“ mache es ihn auch, wenn Forschung sich in eine konkrete Anwendung übersetze und Industriepartner „happy“ seien. Herr Timme wertet Kooperationen mit Industrieunternehmen insofern nicht als eine Art defizitäre, primär auf das instrumentelle Ziel der Drittmitteleinwerbung ausgerichtete Forschung ab. Aus seiner Perspektive sind solche Kooperationen vielmehr die Voraussetzung, damit sich eines der „Features“ seiner Arbeitsgruppe am Ende in einem Produkt wiederfinden könne. Optimalerweise bauten Grundlagenforschung und Anwendungsbezug aufeinander auf:

\footnotetext{
„Also, wir machen wirklich so Grundlagenforschung, ganz crazy Sachen, und wenn da mal was den Weg gegangen ist in Richtung Anwendung, also die Geschichte, das war Thema meiner Bewerbungsvorlesung, wo ich hier zum Vorsingen war im Kolloquium. Und das ist natürlich auch eine besondere Befriedigung, weil ich gesagt habe, dieses Thema, das will ich hier in [Name Uni] hochziehen, und das wollen wir beforschen von der DFG, es hat angefangen mit einem DFG-Projekt dann, bis hin zur Anwendung. Und genauso ist es passiert. Also das ist sehr schön, wenn man so einen Bogen dann auch mal hinkriegt. Das ist nicht häufig. Aber, wenn es mal klappt über die Jahre, ist das schön.“
} 
Insbesondere in dieser Äußerung wird deutlich, welche Relevanz der Anspruch für Herrn Timme hat, einen „Bogen“ von der Forschung in die Anwendung ,hinzukriegen".

Das von Herrn Timme verwendete Bild des „Bogens“ ließe sich aber ebenfalls auf sein Ziel übertragen, seine NachwuchswissenschaftlerInnen auf ihrem Karriereweg zu fördern. Denn im Sinne des schon erwähnten „Meister-Lehrling-Verhältnisses" betont er gerade auch die Vorteile, die sich für seine Mitarbeitenden aus der Verbindung von Grundlagen- und anwendungsbezogener Forschung ergäben. So sei es für ihren weiteren Weg wichtig, ,nicht nur irgendwo im Elfenbeinturm rumgeforscht“ zu haben und zu wissen, ,was ein Meilenstein, was ein Projekt-Bericht" ist. Zudem seien Auszeichnungen wie ein Preis für Technologietransfer „Gold wert“ für sie:

\begin{abstract}
„Also ich brauch das nicht. [...] Mich freut so eine Urkunde wie die, wo zwei Mitarbeiter noch mit draufstehen. Das freut mich. Weil da kann noch was passieren. Die haben noch einen Lebenslauf vor sich, also da würde ich mich freuen, zu irgendeiner Antrittsvorlesung mal von einem von denen eingeladen zu werden. Das wäre so ein richtiger Glücksmoment. Also das sind so Ziele, die ich habe, neben diesem Geld einwerben.“
\end{abstract}

Hier spiegelt sich deutlich die Karrierephase von Herrn Timme wider. Er selbst „brauche“ solche Auszeichnungen nicht mehr, um weitere berufliche Ziele zu erreichen. Eine wichtige Anerkennung wäre es für ihn hingegen, wenn ,die eigenen Gewächse“ erfolgreich ,ihren Weg“ - etwa bis zur eigenen Professur - gingen, womit Herr Timme wiederum sein Rollenverständnis eines akademischen Mentors und Wegbegleiters betont. Das Ziel wäre erreicht, so ergänzt er im Anschluss, wenn sich aus der einstigen Meister-Lehrlings-Beziehung ein berufliches Verhältnis ,,auf Augenhöhe“ entwickle. Damit thematisiert er eine Ebene der persönlichen Bewährung und Anerkennung, die sich kaum in den quantifizierenden Bewertungen der professoralen Lehr- und Forschungsleistungen abbilden lässt.

\title{
Fazit
}

Ungeachtet dessen können wir aber zunächst festhalten, dass Herr Timme keine grundsätzlichen Probleme mit der quantifizierenden Logik universitärer Leistungsbewertungen hat. So bezeichnet er Kriterien wie Drittmittel als sinnvolle „Metrik“ und sieht auch in standardisierten Evaluationen ein durchaus geeignetes Instrument für die Qualitätssicherung/-verbesserung in der Lehre. Ein Grund für diese Perspektive dürften seine beruflichen Erfahrungen in der Industrie sein, aufgrund derer Herrn Timme ein Controlling oder Leistungsvergleiche über bestimmte Kennzahlen nicht fremd sein werden. Darüber 
hinaus ist es aber auch seine Disziplin, die ihm eine positive Aneignung der organisationalen Bewertungsmaßstäbe erleichtert. Die Einwerbung von Drittmitteln gehört für ihn als Nachrichtentechniker zu den normalen Voraussetzungen ,guter ${ }^{6}$ Forschung, sodass die Bewertung seiner Leistungen über dieses Kriterium erst einmal nicht mit alternativen Ansprüchen konfligiert. Die im Rahmen seiner Berufungsverhandlung konkret festgelegte Summe erschien Herrn Timme zwar damals als „,sehr große Zahl“, bei der er „,nicht gewusst“ habe „ob [er] das schaffe“. Dass die Universitätsleitung grundsätzlich Drittmittel von ihm erwartet, bedeutet für ihn aber weder „Druck“ noch „Fremdbestimmung“: Er wolle diese Mittel ,ja auch“, um seine Projekte realisieren zu können.

Er nimmt dieses für universitäre Leistungsbewertungen wichtige Kriterium somit nicht im Sinne eines konstruierten „Normalitätsbereiches“ (Heintz 2010, S. 165) wahr. Hinzu kommt, dass ihm seine Drittmittelprojekte seither - er werbe „deutlich mehr“ ein, als von ihm erwartet wurde - verschiedene Handlungsspielräume verschaffen: neben individuellen Gehaltszulagen die Möglichkeit, die eingeworbenen Mittel in eher unternehmerischer Weise frei verwenden zu können.

Obgleich Herr Timme gleich zu Beginn des Interviews diese Vorteile betont, lässt sich bei ihm jedoch nicht von einer alleinigen Orientierung an jener Anreizlogik sprechen, die mit NPM und Leistungsbewertungen auf die Universitäten übertragen wurde. Denn er sieht einen entscheidenden Mehrwert seiner eingeworbenen Drittmittel darin, unabhängiger von organisationalen Zielen und der Bewertung seiner „Performance“ durch ,irgendein Gremium“ zu sein. ${ }^{5}$ Dass Herr Timme wiederholt davon spricht, durch seine Drittmittel-Projekte weitgehend ,in Ruhe gelassen“ zu werden, könnte insofern irritieren, als er mit seiner Kombination aus Grundlagenorientierung und Anwendungsbezug im Einklang mit dem etablierten Forschungsverständnis seiner Universität agiert. Wovon sich Herr Timme aber abgrenzen möchte, sind übergeordnete, vonseiten des Rektorats angestoßene Profilbildungsprozesse, die dem Ziel einer möglichst guten Position der Universität im interuniversitären Wettbewerb dienen. Zum einen widersprechen diese Prozesse seinem Verständnis, nach dem außergewöhnliche und von der Scientific Community anerkannte Forschungsleistungen einzelner ProfessorInnen und ihrer Arbeitsgruppen vorrangige Bedeutung haben sollten. Zum anderen

\footnotetext{
${ }^{5}$ In einem weiteren Verständnis kann das freilich eine gesteigerte Form des neoliberalen „unternehmerischen Selbst“ (Bröckling 2007) sein, bei dem der Fremdzwang von NPM zum internalisierten Selbstzwang geworden ist. Es kommt darauf an, ob Herr Timme Forschungen, die ihm sonst auferlegt worden wären, ,freiwillig' betreibt oder aber eigensinnige Forschungsagenden verfolgt.
} 
konfligiert die Einbindung in universitäre Profilbildungsprozesse mit seinem Anspruch, weiterhin und auf kurzen Wegen - als „Meister-Mentor“ - an der Forschung seiner Arbeitsgruppe teilhaben zu können.

Mit seiner Entscheidung, sich zukünftig nicht mehr in entsprechenden Gremien zu engagieren oder an größeren Forschungsverbünden zu beteiligen, behauptet Herr Timme somit auch identitätsbezogene Ansprüche an seine Forschung. Ihm geht es nicht allein um den strategischen Nutzen des individuellen Antragserfolges. Seine Entscheidung gegen eine weitere Beteiligung an Verbundanträgen und universitärer Profilbildung ist ferner mit Blick auf seine Karrierephase und sein Standing an der Universität zu sehen. Nach fast zehn Jahren, in denen er als Professor bereits erfolgreich Drittmittel einwerben konnte, sieht er sich nicht mehr dem Druck ausgesetzt, sich noch bewähren zu müssen. Zudem verfolgt er keine Karrierepläne, für die ihm die Leitung eines neuen Forschungszentrums oder einer Forschungsgruppe wichtige Vorteile verschaffen könnte.

\subsection{Frau Lange: Zwischen Anpassung an Leistungsbewertungen und Behauptung eigener Ansprüche (LIT3)}

Für Frau Lange, eine W3-Professorin für Neuere Deutsche Literatur, verbinden sich die universitären Leistungsbewertungen mit mehr Ambivalenzen und wahrgenommenen Gefährdungen ihrer eigenen Ansprüche an Lehre und Forschung als für Herrn Timme. Eine nicht unwichtige Rolle spielt dabei ihre Fachdisziplin, in der die Forschungsarbeit weniger von eingeworbenen Drittmitteln abhängt und alternative Bewertungsmaßstäbe wie etwa Monografien existieren. Hinzu kommt, dass Frau Lange in der Position einer relativ neu berufenen Professorin unter einem Bewährungsdruck steht und es für sie insofern auch darum geht, mit ihren Leistungen von der Organisation wie auch ihren KollegInnen anerkannt zu werden. Beides trägt dazu bei, dass sich Frau Lange intensiv mit der universitären Bewertungsordnung auseinandersetzt. Anders als Herr Timme ist sie noch eher auf der Suche nach dem richtigen Weg zwischen Anpassung und Behauptung eigener Ansprüche. Eine wichtiger Punkt ist für Frau Lange dabei die zukünftig ,richtige' Gewichtung von Forschung und Lehre.

\section{Deutung der neuen Governance: Mehr Druck und Kontrolle}

Dass Frau Lange die neue Governance als deutlich ambivalent wahrnimmt, zeigt sich bereits in der Eingangssequenz des Interviews. So antwortet sie auf die 
noch eher allgemeine Frage nach ihrer Wahrnehmung universitärer Wandlungsprozesse:

„Ich hab ja hier angefangen schon mit Ziel- und Leistungsvereinbarungen und so. Insofern kann ich schlecht das mit einem Zustand davor vergleichen, aber klar, ich mein, das ist vielfältig. Dass man schon das Gefühl hat, zusätzlich zu der ja normalerweise in unserem Berufsstand sehr weitgehend vorhandenen intrinsischen Motivation gibt's jetzt einfach dieses vielfältige, auch sehr differenzierte Regelsystem irgendwie, dass das, was man so tut, beobachtet wird, klassifiziert wird, mit Punkten versehen wird und so weiter. [...] Also ich finde schon, gerade auch wenn man's vergleicht mit den Kollegen in den USA oder so, dass der Druck hier enorm ist, man ist eingespannt in ein unglaublich enges Korsett. Was schade ist. Denn ich will nicht sagen, dass dadurch schlechtere Forschung gemacht wird, aber es wird einfach doch viel produziert, auch viel immer im Hinblick auf bestimmte Anforderungen, die man natülich zunehmend internalisiert.“

Frau Lange kommt gleich zu Beginn auf beide Seiten der Leistungsbewertungen zu sprechen: zum einen auf den organisationalen Kontrollanspruch, der sich in einem „differenzierte[n]“ Klassifikationssystem und der Praxis widerspiegle, die Leistungen der ProfessorInnen ,mit Punkten [zu] versehen“; zum anderen auf die Folgen für die Bewerteten, welche sich durch den ,enormen“ Druck und das „unglaublich enge[] Korsett“ allmählich an die aus den Leistungsbewertungen resultierenden „Anforderungen“ anpassten.

Solche Prozesse der „Internalisierung“ reflektiert Frau Lange auch mit Blick auf ihre eigene Person. So fährt sie unmittelbar mit folgender Äußerung fort:

\begin{abstract}
„Als ich anfing, war mir manches noch nicht so klar. Nachdem ich das erste Mal die LOM-Abrechnung ${ }^{6}$ am Ende des Jahres gemacht hatte, hab ich dann manche Dinge auch anders wahrgenommen. Also gerade zum Beispiel auch Prüfungseinsatz. Man weiß dann plötzlich, dass nen Zweitgutachten eigentlich nichts zählt, und bei mir ist es persönlich so, dass es gar nicht unbedingt dann dazu führt, dass ich das nicht mehr mache oder das nur noch provisorisch lese oder so, was man wahrscheinlich tun sollte. Ja, aber das kann ich irgendwie nicht. Ich hab dann immer das Gefühl, ich muss es wirklich lesen und wirklich begutachten und so, aber man wird frustriert. Man hat das Gefühl, was verbringe ich jetzt hier die ganze Zeit damit, das darf ja nicht sein. Ich krieg dafür ja gar nichts. Also das sind so Effekte, die nen bisschen unschön sind.“
\end{abstract}

\footnotetext{
${ }^{6}$ Frau Lange bezieht sich hier auf das in Kap. 1 erläuterte Instrument der leistungsorientierten Mittelvergabe, welches an ihrer Universität offensichtlich bis zur Ebene der einzelnen ProfessorInnen angewendet wird.
} 
Den ersten näheren Einblick in die bei der leistungsorientierten Mittelvergabe (LOM) zugrunde gelegten Leistungskriterien beschreibt sie als quasi einschneidenden Moment. Sie musste erfahren, dass ihre Sorgfalt - hier in der Rolle der Zweitgutachterin - „eigentlich nichts zähl[e]“. Frau Lange deutet ihre hohen Selbstansprüche insofern im Sinne eines Nachteils und spricht explizit davon, durch die fehlende Anerkennung „,frustriert“ zu werden.

\title{
„Bologna“ und „Employability“: Keine Bedrohung ,guter 'Lehre
}

Dass Frau Lange die Entwertung ihrer Leistungen zu Beginn des Interviews anhand eines Beispiels aus der Lehre bzw. Nachwuchsförderung illustriert, ist alles andere als zufällig. Denn für sie handelt es sich hierbei um sehr positiv besetzte, für ihre professorale Identität wichtige Aufgaben:

\begin{abstract}
,[M]ir macht Lehre auch sehr viel Spaß, muss ich sagen, auch Betreuung, das ist so mit das Befriedigendste an diesem Job, finde ich, dass man sieht, dass man bestimmte Anregungen gibt und die das dann selber weiterentwickeln, und wenn dann jemand plötzlich ne ganz eigene Masterarbeit aus solchen Themenfeldern entwickelt oder vielleicht dann sogar weiter promovieren will, das find ich schon sehr, sehr toll. Und da nehme ich mir auch viel Zeit $\mathrm{zu}$, die zu betreuen und Gutachten schreiben, dass die weiterkommen und so weiter. Gehört für mich eigentlich auch zur Lehre alles mit dazu."
\end{abstract}

Frau Lange bezieht sich mit ihren positiven Deutungen ebenso auf reguläre Lehrveranstaltungen. Wichtig ist hierbei auch, dass sie nicht zu jenen „Bologna“KritikerInnen aus ihrer Disziplin gehört, die die Studienreform mit einem Qualitätsverlust der universitären Lehre verbinden. Sie sieht mit Blick auf die Einführung von Bachelor- und Masterstudiengängen zwar durchaus kritische Punkte. So hält sie es für fragwürdig, dass ein Bachelorabschluss in ihrer Disziplin bereits als eigenständige Qualifikation gelten könne. Da sich in den entsprechenden Qualifikationsarbeiten nicht selten der relative Anfängerstatus der Studierenden widerspiegle, erscheint ihr ein Masterabschluss als notwendig. Gleichwohl bedient sich Frau Lange keiner generellen Krisenmetaphorik:

„[I]ch muss schon sagen, man merkt den MA-Leuten an, dass sie schon mal nen Examen gemacht haben, dass man da einfach schon mal an nem bestimmten Punkt ansetzen kann. Und so, das wissenschaftliche Arbeiten ist oft, die BA-Arbeiten sind oft nen bisschen schwierig, also wo man dann einfach merkt, so manche Sachen sind halb verstanden, aber manches ist noch so ungar. Und trotzdem ist es eigentlich so, gerade auf dem MA-Niveau, dass viele doch eine ganze Menge, erstaunlich viel mitgenommen haben und das selbstständig dann auch weiterentwickeln können. Ich bin da nicht so pessimistisch.“ 
Auch die u. a. im medialen Diskurs prominente Forderung nach einer stärkeren Orientierung der Lehre an der „Employability“ der Studierenden deutet sie nicht im Sinne einer grundsätzlichen Bedrohung für ihr Fach. Frau Lange geht von keinem universitären Bildungsideal aus, demgemäß die Lehre gegenüber jeglichen Zweck- oder Nutzenerwägungen zu schützen sei. Da sie und ihre KollegInnen ,natürlich“ nicht allein „Wissenschaftler ausbilde[te]n“, sei es vielmehr angemessen, das eigene Fach auch hinsichtlich seiner Anschlussfähigkeit an den Arbeitsmarkt zu betrachten:

„,[U]nd ich bin auch der Meinung, dass man sich auf diese Diskussion einlassen muss. Also es gibt ja auch manche Leute, die sagen: ,Wir diskutieren gar nicht mit euch, wenn ihr nur die Praxisorientierung wollt.' Sondern ich denke, es gibt gute Argumente dafür, warum Geisteswissenschaften erstmal grundsätzlich Denkweisen, auch Formen der Kritik, der Analyse, des Verstehens, des systematisierten Verstehens und so weiter vermitteln, ohne dass man das sofort ankoppeln muss an eine Berufsperspektive. Ja. Und das kann man und das sollte man auch immer wieder so erklären.“

Im Gegensatz zur abwehrenden Haltung einiger FachkollegInnen plädiert Frau Lange dafür, den Mehrwert eines geisteswissenschaftlichen Studiums in selbstbewusster Weise zu behaupten. Schließlich handele es sich bei einem „systematisierten Verstehen“ oder der Anwendung analytischer „Denkweisen“ auch um nützliche, ,,in der Wissensgesellschaft unabdingbar[e]“, Kompetenzen.

Für Frau Lange besteht insofern kein Widerspruch zwischen einer weiterhin anspruchsvollen Lehre und der Reflexion eines möglichen Nutzens für Berufsfelder jenseits der Wissenschaft. In dieser eher gelassenen Sichtweise sieht sie sich nicht zuletzt durch die Studierenden bestätigt. Denn bei ihnen gehe das Bemühen um eine konkretere berufliche Perspektive nicht selten mit einer hohen Motivation für das Fach einher:

„Und die wollen was mit diesem Studium! Also ich mach insgesamt wirklich sehr positive Erfahrungen, viele machen das ganz toll, viel besser, als ich das früher gemacht habe, also sich gut zu organisieren mit Jobs nebenher, die aber zum Teil auch so gewählt sind, dass sie gut schon ineinandergreifen und im Grunde daraus dann auch schon so ne Berufsperspektive sich entwickelt. Das ist ja bei Geisteswissenschaften auch immer schwierig. Wenn man jetzt nicht Lehramt studiert, ist das in dem Sinne keine Berufsausbildung, aber das machen viele wirklich ganz klasse, also sind sehr fokussiert, sehr interessiert.“

Frau Lange zeichnet alles andere als das Bild einer zu angepassten Studierendengeneration und ergänzt an anderer Stelle, dass viele der Studierenden die 
Bereitschaft zeigten, sich intensiv auf Themen und „ganz viel Lektürepensum“ einzulassen. Insofern sieht sie ihre Ansprüche an ,gute " Lehre weder durch die „Bologna"-Reformen noch durch die Haltung der Studierenden gefährdet. Anders formuliert, geht Frau Lange bei ihren Deutungen weniger von studentischen Defiziten als von der Prämisse aus, sich an einer ,,veritablen Studierendenschaft“" (Liebeskind 2011, S. 208) orientieren zu können, die den Kern der Wissenschaft erfassen und ,echtes' Interesse für das eigene Fach aufbringen könne. Dies zeigt sich auch, wenn sie konkreter auf ihre Lehrziele und -veranstaltungen zu sprechen kommt.

Frau Lange betont zwei Lehrziele, die wir bereits bei vielen der in Kap. 3 zitierten ProfessorInnen aus den Geistes- und Sozialwissenschaften gesehen haben. Zum einen geht es ihr darum, einen gemeinsamen Reflexionsprozess zu fördern, in dem die Studierenden eine aktive Rolle einnehmen. Zu vermeiden sei „so eine hierarchische Situation, da macht einer irgendwie nen Unterricht und dann schreibt man eine Hausarbeit“. Zum anderen ist Frau Lange eine ,enge Verknüpfung von Forschung und Lehre“" wichtig, weil.

„,man eben gerade dann immer wieder auch Dinge so vermitteln kann, dass sie noch nicht zu Ende gedacht sind. Dass man alle dazu einlädt, mitzumachen, da weiter zu denken und Sachen zu entdecken. Das, glaub ich, ist wichtig, dass man nicht das Gefühl hat: ,So jetzt hab ich hier so meine Routine und meinen Kanon und so kann es jetzt die nächsten zehn Jahre bleiben.““

Gerade durch die Integration von Forschung und Lehre ergäben sich für beide Seiten Vorteile: Die Studierenden würden durch die vermittelte Offenheit dazu „eingeladen“, Neugierde und eigene Ideen zu entwickeln. Und sie als Lehrende vermeide so, routiniert auf einen festen Kanon zurückzugreifen und eigene Entwicklungsmöglichkeiten zu sehr zu begrenzen.

Frau Lange gehört dabei zu jenen ProfessorInnen, die positive Lehrerfahrungen nicht an ein bestimmtes Vorwissen oder Niveau der Studierenden knüpfen. Entscheidender ist für sie, dass sich die Teilnehmenden ihrer Seminare auf neue Betrachtungsweisen literarischer Werke einlassen und ein Verständnis dafür bekommen,

„,dass man erst mal so einen Text für sich anguckt, wie werden da Dinge konstituiert, also wie wird irgendetwas, ein Sinn, behauptet, aber wie wird im gleichen Text sozusagen dieser Sinn auch unterlaufen, wie entsteht überhaupt so etwas wie Bedeutung, wie wird Welt konstruiert und mit welchen Strategien kann aber auch sozusagen die Veränderbarkeit von kulturellem Wissen erkannt werden?“ 
Neben dem Lehrziel einer analytisch-wissenschaftlichen Perspektive geht es Frau Lange gerade auch darum, einen persönlichen Bezug der Studierenden zu den jeweiligen Inhalten zu fördern. Als Beispiel für eine besonders "schöne“ Erfahrung beschreibt sie etwa eine "sehr ergreifende Hausarbeit", in der eine Lehramtsstudentin

„das dann so eingerahmt hat in ihre persönlichen Erfahrungen. Das fand ich dann schon sehr berührend, was die mitgenommen hat in diesem Seminar, wie sie dann doch das einfach auch so für sich produktiv gemacht hat. Und jetzt zu wissen: Okay, die wird demnächst Deutschlehrerin [...], das ist schon, das ist toll.“"

Frau Lange stellt insgesamt weniger ihre eigene vermittelnde oder anleitende Rolle als die individuelle und teils kreative Aneignung der Inhalte durch die Studierenden in den Mittelpunkt. Auch sie profitiere von den Studierenden, wenn diese etwa neue Themen oder Genres wie Graphic Novels in die Veranstaltungen einbrächten und ihrerseits ,innovativ“ agierten. Frau Lange betont insofern das Qualitätsmerkmal einer Wechselseitigkeit in der Lehre. ${ }^{7}$

Als eher unbefriedigendes Lehrformat erlebt sie entsprechend Vorlesungen. Sie könne zwar auch in diesem Rahmen aktuelle wissenschaftliche und gesellschaftliche Diskurse aufgreifen. Schwerer wiegt für sie jedoch der Nachteil, in eine zu große Distanz zu den Lern- und Reflexionsprozessen der Studierenden zu geraten:

„Ich versuch dann immer so interaktive Elemente mit einzubauen, dass ich einfach zwischendurch mal nen Gedicht hab oder so, und dann frag ich einfach in den Raum. [...] Ich finde diese Form Vorlesung nicht so befriedigend, weil man von den Studierenden zu wenig mitbekommt, man kriegt weniger mit, was bei denen ankommt, was deren Fragen sind.“

Gerade im Vergleich zu den Deutungen von Herrn Timme zeigt sich, dass diese Form der Lehre für sie nur wenig sinnstiftend ist. Während Herr Timme von seiner eigenen Begeisterung ausging, die sich im Optimalfall auf die

\footnotetext{
${ }^{7}$ Frau Lange macht eine solche Wechselseitigkeit nicht an einem möglichst ebenbürtigen Kenntnisstand von Lehrender und Studierenden fest. Damit unterscheidet sie sich von jenem Literaturwissenschaftler, dessen Deutungen wir in Abschn. 3.2 dargestellt haben und der von dem Ideal einer ,geteilten Souveränität“ (LIT1) im Umgang mit anspruchsvollen Texten ausgegangen war. Für Frau Lange ist entscheidender, dass beide Seiten einen Bezug zu den Themen finden und auf Basis eines geteilten Interesses ein Austausch entsteht.
} 
Studierenden übertrage, steht für Frau Lange die Asymmetrie des Settings im Vordergrund. Da sie ,gute“ Lehre vor allem als wechselseitigen Prozess definiert, kann sie sich ein „Bild der Lehre als Bühne“ (Liebeskind 2011, S. 203) nicht positiv zu eigen machen.

Evaluationen und didaktische Weiterbildung: Keine wirkliche Anerkennung der Lehre

Frau Lange kommt im Kontext der Vorlesungen jedoch auch auf den mit der Vorbereitung verbundenen ,irren Aufwand“ zu sprechen und deutet damit bereits ein für sie zentrales Problem an: das zu geringe Zeitbudget, das für eine wirklich substanzielle Erfüllung der Lehraufgabe zur Verfügung stehe. Einige ihrer älteren KollegInnen investierten mittlerweile weniger Zeit und Mühe in die Vorbereitung ihrer Veranstaltungen. Aus Sicht von Frau Lange ist dies zwar nicht per se kritikwürdig, da ein routinierterer Umgang mit der Lehre keinen zwangsläufigen Qualitätsverlust bedeute:

„,[D]ie haben ihre Vorlesungen, die haben sie schon ein paar Mal gehalten, die sind da. Das ist dann auch nicht mehr so viel Aufwand, die nochmal zu halten. Das heißt ja nicht, dass die dann schlechter ist."

Sie selbst hält aber an ihrem Anspruch fest, Vorlesungen und Seminarthemen stetig zu aktualisieren. Da ihr darüber hinaus eine verlässliche Betreuung des wissenschaftlichen Nachwuchses wichtig ist und sie auch schon interessierte Studierende bei ersten Publikationen - etwa durch ein teils aufwendiges Redigieren ihrer Artikel - unterstützen wolle, sieht sie sich mit einer Situation der Überlastung konfrontiert, die sie derzeit kaum aufzulösen oder abzumildern vermag. So resümiert Frau Lange, dass ihr Alltag durch „ein Gerenne“ bestimmt sei. Ursächlich hierfür sei insbesondere ein ,viel zu hohes Lehrdeputat“, welches sie und ihre KollegInnen bewältigen müssten.

Mit ihrem Verweis auf das Lehrdeputat markiert Frau Lange einen Bedarf an grundsätzlicheren Veränderungen für eine Verbesserung der Lehre. Entsprechend kritisch sieht sie den organisationalen Fokus auf Instrumente wie Evaluationen oder mögliche didaktische Weiterbildungsangebote. Diskrepanzen zwischen dem organisationalen Anspruch, die universitäre Lehre zu verbessern, und dessen tatsächlicher Umsetzung zeigten sich für sie bereits im Kontext ihres Berufungsverfahrens. Als Angehörige der jüngeren ProfessorInnengeneration habe sie zwar zusätzlich auch ihre Kompetenzen in der Lehre unter Beweis stellen müssen. Sie sei dabei aber auf ein relativ unverändertes organisationales Setting getroffen: 
„[E]s ist ja, schon als ich mich beworben hab, überall war es eigentlich, ich hatte dann immer so ein Lehrkonzept geschrieben, aber eigentlich ist es absurd, weil die, die sich das dann angucken in den Kommissionen, haben auch keine hochschuldidaktische Ausbildung, also jeder macht so irgendwie, wie er meint, und dann plötzlich wird man begutachtet in der Lehre. [...] Es ist zwar ganz nett dieses Bemühen, das stärker zu etablieren, aber in der Praxis spielt es zu wenig eine Rolle; und vor allen Dingen, es gibt kein Zeitbudget dafür. Es wird dann irgendwie erwartet, dass man das noch so zusätzlich macht, aber es wird nicht wirklich dem Ganzen nochmal Raum gegeben, und dann kann es natürlich auf die Dauer auch nicht so gut funktionieren."

Frau Lange kritisiert nicht, dass ihr Lehrkonzept überhaupt begutachtet und sie insofern auch als Lehrende bewertet wurde. Das Kritikwürdige ist für sie vielmehr, dass die bewertende Instanz selbst über keinen professionellen Maßstab verfügte.

Wichtiger sei aber noch, dass aus dieser Form der Bewertung danach im Grunde nichts mehr folgte. Aus Frau Langes Sicht wird die Verantwortung für eine Verbesserung der Lehre nach Abschluss der Berufungsverfahren im Wesentlichen individualisiert, während die strukturellen Probleme - vor allem: das fehlende Zeitbudget - bestehen blieben. Diese Haltung der Organisation spiegle sich schon in der Anwendung von Instrumenten wie Lehrevaluationen wider. So werde die Evaluation an ihrer Universität nicht etwa ,regelhaft und verpflichtend", sondern je nach Interesse und Bedarf der ProfessorInnen durchgeführt. Frau Lange deutet diese Vorgehensweise nicht als Entlastung von organisationaler oder studentischer Leistungskontrolle, sondern als Ausdruck einer nur wenig ernsthaft betriebenen Qualitätsverbesserung der Lehre.

Sie selbst nimmt Evaluationen für sich als hilfreiches Instrument wahr und habe schon früher „öfter mal selber so Fragebögen entwickelt“. Lehrevaluationen ermöglichten schließlich, die unweigerlich wachsende Distanz zu der studentischen Perspektive zumindest etwas überbrücken zu können:

„Und ich finde auch, besonders je älter man wird, also als ich so angefangen hab zu unterrichten, fühlte ich mich den Studierenden auch ganz nah, konnte ich mir noch eher vorstellen, wo kommen die so her, was haben die für Erwartungen, was geht in denen vor? Je länger man selbst drin ist, ist man erstens natürlich irgendwann eine andere Generation, ganz anders geprägt, aber man hat natürlich auch selbst von seiner eigenen wissenschaftlichen Arbeit so viel im Kopf, was man dann im Einzelnen vielleicht nicht ausreichend expliziert oder herleitet, so dass es, glaube ich, sehr wichtig ist, bei den Studierenden immer wieder nachzuhaken, wo kommt ihr her, könnt ihr hier folgen, könnt ihr da folgen, was hättet ihr gerne sonst noch?“ 
Frau Lange sieht in der studentischen Bewertung also eine hilfreiche Basis, um ihre Lehre im Sinne der Lern- und Entwicklungsprozesse der Studierenden zu gestalten. ${ }^{8}$

Über diese freiwillig durchgeführten Evaluationen hinaus gebe es zwar ein universitäres Angebot an didaktischen Weiterbildungsveranstaltungen. Wie den meisten der ProfessorInnen fehle aber auch ihr hierfür die Zeit, „obwohl es vielleicht interessant und hilfreich “ wäre. Auch ihre eigene Initiative, regelmäßige Treffen „unter den Kollegen“ zum Austausch über Lehrerfahrungen zu etablieren, scheitere an den geringen zeitlichen Ressourcen der potenziell Beteiligten und stoße letztlich eher auf die Reaktion: ,[N]icht noch eine Sitzung, nicht noch irgendwas."

Gerade weil die Lehre für Frau Lange weit mehr als eine notwendige Pflicht ist, erlebt sie diese strukturell bedingten Grenzen als dilemmatisch. Einen gewissen Vorbildcharakter schreibt sie in diesem Kontext den USA zu, wo die universitäre Lehre eine stärkere und institutionalisierte Förderung erfahre:

\begin{abstract}
„Zum Beispiel, man kann da ein Stipendium gewinnen, das finde ich ja ganz toll, so diese Vorstellung, wenn man das bekommt, dann kriegt man lehrfrei ein Semester und hat in dieser Zeit die Gelegenheit, eine Vorlesung, die man schon mal gehalten hat, auf didaktische Aspekte und so weiter nochmal komplett zu überarbeiten. Das finde ich so klasse, also diese Vorstellung, man braucht Zeit, um gute Lehre zu entwickeln.“
\end{abstract}

Entscheidend ist für Frau Lange, dass , gute" Lehre in den USA nicht als abgeleitetes Ergebnis oder Nebenprodukt ,guter' Forschung betrachtet wird. Mit dem von ihr angeführten „Stipendium“ werde schließlich anerkannt, dass die Überarbeitung einer Vorlesung ähnlich wie die Forschung zu einem bestimmten Thema zeitliche Freiräume erfordere. Demgegenüber beschreibt Frau Lange ihre eigene Situation über einen subalternen Rang der Lehre. So habe sie nur im Rahmen eines Forschungsfreisemesters die Möglichkeit gehabt, einen „richtig umfangreichen tollen Reader" für eine Veranstaltung zu erstellen. Insgesamt nehme sie eine zunehmende Hierarchisierung zugunsten drittmittelstarker ProfessorInnen und durch den „Drittmittel-Druck“ an „vielen Unis“ die Entwicklung zu einer Art „Zwei-Klassen-Gesellschaft“"wahr. Da gebe es jene,

\footnotetext{
${ }^{8}$ Insofern ergänzt sie auch, dass Evaluationen nicht am Semesterende, sondern „,mittendrin“ durchgeführt werden sollten, um noch etwas ,,verändern“ zu können.
} 
„die von allem befreit sind und sich nur noch um die exzellente Forschung bemühen, und die anderen, die eben lehren und da aus diesem Hamsterrad auch nicht mehr richtig rausgucken, um dann selber noch größere Anträge zu stemmen.“

Frau Lange bezieht die skizzierte Privilegierung drittmittelstarker ProfessorInnen hier eher allgemein auf verschiedene Universitäten. Wir werden später allerdings noch darauf zurückkommen, wie Frau Lange solche Prozesse der Hierarchisierung und den wachsenden Konkurrenzdruck an ihrer eigenen Fakultät wahrnimmt. An dieser Stelle kann aber bereits festgehalten werden, dass die beschriebene Belohnung von Forschungsleistungen zu ihrem Eindruck beiträgt, als Lehrende kaum organisationale Anerkennung zu erfahren.

\section{Forschung: Fragile Anerkennung und informeller Anpassungsdruck}

Für Frau Lange ergeben sich auch aus den an die Forschung angelegten Bewertungskriterien deutliche Ambivalenzen. So kritisiert sie, dass die Einwerbung von Drittmittelprojekten eine immer wichtigere Rolle spiele und eine zunehmend am ökonomischen ,Pay Off‘ orientierte In-Wert-Setzung von Forschung erfolge:

„Also es wird die ganze Zeit erwartet, dass man Drittmittelanträge schreibt natürlich; und auch, das ist klar, die Unis brauchen die Mittel, und dann wird uns das sozusagen so verkauft, als ob es keine wertvolle wissenschaftliche Tätigkeit gäbe ohne dieses Einwerben von Drittmitteln [...].“

Aus Frau Langes Sicht agieren die „Unis“ zum einen strategisch, indem sie die Drittmittelhöhe als Bewertungskriterium ,verkauften“, obwohl es ihnen vor allem um ihre Finanzierungsbasis gehe. Zum anderen fänden disziplinäre Unterschiede zu wenig Berücksichtigung, sodass geisteswissenschaftliche Forschungsleistungen, die häufiger ohne Drittmittel auskämen, als entsprechend weniger „,wertvoll“ eingestuft würden. Für Frau Lange scheint somit keine wirklich ernsthafte Auseinandersetzung mit der Frage stattzufinden, wie ,gute' Forschung tatsächlich in angemessener und gerechter Weise bewertet werden könnte.

Dies bestätigt sich, wenn sie im Weiteren auf die organisationale Bewertung von Publikationen zu sprechen kommt, die sich aus ihrer Sicht zu sehr an der Publikationskultur naturwissenschaftlicher Disziplinen orientiere:

\footnotetext{
„Also wir haben diesen Impact-Faktor, diese ganzen Berechnungen, was die Naturwissenschaften haben, so nicht, und das ist auch nicht sinnvoll. Und jetzt aber so zu tun, als ob man da eine Qualitätssicherung durch dieses Moment Peer Review einführen kann, die aber intern in der Fachkultur sich so gar nicht etabliert hat oder nur sehr teilweise, das ist dann einfach, würde ich sagen, auch an der Fachkultur
} 
vorbei. Also ich hab eine Kollegin, die hat sofort, als das rauskam, wollte Anträge schreiben, DFG-Anträge. Hat sie gesehen: ,Aha. Das ist jetzt so. So, jetzt muss ich also gucken, dass ich meinen Aufsatz in den und den Zeitschriften platziere, die als peer-reviewed gelten. 'Und ich weiß nicht, ob die Aufsätze dadurch jetzt unbedingt besser geworden sind. Natürlich nicht, ja. Also das ist genau der gleiche Aufsatz."

Frau Lange verweist hier auf Diskrepanzen zwischen den angelegten Bewertungskriterien und ihrer Disziplin, aber auch auf Anpassungsprozesse, die sie bereits in ihrem kollegialen Umfeld beobachte. Offen ist damit allerdings noch, inwieweit sie die organisationalen Leistungskriterien auch als Gefährdung ihrer eigenen Ansprüche an ,gute' Forschung wahrnimmt.

Wichtig ist, dass sich Frau Lange nicht im Sinne einer klassischen Individualforscherin positioniert. Sie betont zwar durchaus die Bedeutung ihres individuellen intrinsischen Bezuges zu bestimmten theoretischen Perspektiven und Fragestellungen. So komme sie „immer wieder“ auf die Gegenstände ihrer Qualifikationsarbeiten zurück und finde ihre, ,ganzen Themen immer unheimlich interessant"“. Zugleich grenzt sie sich jedoch explizit vom Bild einer Wissenschaftlerin ab, die gewissermaßen aus sich heraus neue Erkenntnisse schaffe, ohne dabei durch fachinterne und gesellschaftliche Diskussionen inspiriert zu werden:

„,[I]ch bin Teil eines Netzes, und natürlich bin ich jetzt nicht irgendwie genialisch allein auf einer Insel und hab in einer Tour wahnsinnige Ideen, sondern, ohne das $\mathrm{zu}$ wissen oft, ist man beeinflusst, befruchtet durch theoretische Diskussionen, auch durch öffentliche Veränderungen, und das alles so miteinander zu verbinden, dass man dann für uns jetzt auch mit kanonischen Texten, die jeder zu kennen glaubt, neue Dinge tun kann, das ist das, was gute Forschung dann ausmacht.“

Ihre positive Deutung, immer auch „Teil eines Netzes“ zu sein, spiegelt sich ebenso in der Darstellung ihrer Forschungspraxis wider. In Kap. 3 haben wir gesehen, dass einige der LiteraturwissenschaftlerInnen, aber auch StrafrechtlerInnen vor allem von dem Ideal einer, einsamen' Forschung ausgehen. Frau Lange rückt von einem solchen Ideal hingegen eher ab. So resümiert sie mit Blick auf ihre Qualifizierungsarbeiten, dass es „auch schwierig“ gewesen sei, über einen längeren Zeitraum ,wirklich ganz alleine“ und „so für sich selber produzieren“ zu müssen. Aktuell suche sie auf Tagungen oder durch gemeinsame Veröffentlichungen stärker den kollegialen Austausch. Zudem sei sie bereits in ein kleineres Forschungsprojekt eingebunden und plane, sich um weitere Drittmittelprojekte zu bemühen, auf die sie dann sukzessive ,aufsatteln“ könne. Insofern gehört Frau Lange eigentlich zu jenen LiteraturwissenschaftlerInnen, deren Ansprüche an die Forschung nicht per 
se durch solche organisationale Bewertungskriterien gefährdet werden, die auf eine projektförmige und kooperativ vernetzte Forschungsarbeit abzielen. Dennoch ist die konkrete Umsetzung der universitären Leistungsbewertungen für sie mit ambivalenten Erfahrungen verbunden.

Ein wesentlicher Grund ist, dass Frau Lange ihre Forschungsleistungen seitens der Organisation nur teilweise anerkannt sieht. Insbesondere in Bezug auf ihre Publikationen nimmt sie das organisationale Bewertungs- und Punktesystem als nachteilig wahr. Wie folgende Äußerung zeigt, fällt es ihr trotz ihrer kritischen Distanz gegenüber den vorgegebenen Leistungskriterien schwer, sich auch von der Bewertung als solcher zu distanzieren:

„Ich meine LOM, na das ist, man kann sich dem nicht entziehen, also es hat dann einfach, wenn man weiß, wie viel Punkte man für was kriegt. Zum Beispiel ist es so: Man kriegt nur für einen Aufsatz ab zehn Seiten überhaupt irgendwelche Punkte. Ist natürlich schlecht, wenn man jetzt einen Lexikonartikel veröffentlicht, der vielleicht viel mehr Arbeit macht, aber der nur fünf Seiten hat. Das ist dann schade. Dann überlegt man sich womöglich beim nächsten Mal, den überhaupt anzunehmen, weil man dafür ja gar nichts kriegt außer ganz viel Arbeit. Natürlich, das ist jetzt überspitzt, also vielleicht kriegt man von der Community irgendwann mal was zurück, weil man diesen genialen Lexikonartikel verfasst hat. Aber klar, ich mein, das ist halt schon eine Auswirkung."

Obwohl Frau Lange um den Wert ihrer „Lexikonartikel“ für die Scientific Community weiß, kann sie sich nur bedingt der nach „Seiten“ quantifizierenden Bewertungslogik „entziehen“. Ähnlich wie bei dem gleich zu Beginn der Falldarstellung skizzierten Beispiel ausbleibender LOM-Punkte für Zweitgutachten hat das organisationale Belohnungssystem auch in diesem Fall demotivierende Folgen für sie.

Für Frau Lange kommt hinzu, dass durch das forcierte Leistungskriterium von peer-reviewed Publikationen auch ihre Beiträge in Sammelbänden entwertet werden, die oftmals „im Anschluss an Konferenzen“ erschienen und innerhalb ihrer Disziplin eine wichtige Rolle spielten. Gerade wenn sie zudem als Herausgeberin fungiere, bereite ihr die geringe organisationale Honorierung solcher Sammelbände „nicht so gute Laune“. Schließlich machten sie und ihre KollegInnen sich „unglaublich viel Arbeit“, ließen „Dinge nochmal umschreiben“ und täten im Grunde das, ,was Peers auch machen würden“. Im Ergebnis zahle sich dieses Engagement bei der LOM jedoch kaum aus.

Anders stellt sich dies für Frau Lange allerdings mit Blick auf das Kriterium der Drittmittelprojekte dar. Denn in dieser Hinsicht machte sie die Erfahrung, bei 
der LOM eher wider Erwarten „die höchste Belohnung“ für das bereits erwähnte kleinere Forschungsprojekt zu bekommen. Die folgende Äußerung zeigt eindrücklich, dass Frau Lange damit weit mehr als nur den ,Pay Off‘ zusätzlicher Ausstattungsmittel verbindet:

\begin{abstract}
„Und da muss ich sagen, da war ich dann doch irgendwie, nachdem ich vorher immer das Gefühl hatte, keiner interessiert sich dafür, was ich hier rumstrampel mit diesem Projekt, das kann ich gar nicht richtig angeben, weil es ja keine DFG-Mittel sind [...], und dann hat der Fachbereichssprecher halt gemeint: ,Doch: Schreib das doch alles mal da mit dazu. 'Und tatsächlich, sie haben alles anerkannt, und das hab ich dann schon auch, das war dann irgendwie nen Signal: Das ist schon gut und es wird irgendwie geschätzt. [...] Da kommt auch mal etwas zurück. Und sei es jetzt auch nen bisschen auf eine komische Weise quantifiziert vielleicht, aber immerhin.“
\end{abstract}

Das Instrument der LOM bedeutet für Frau Lange in diesem Fall die Chance, mit ihrer Forschung nicht nur von der Scientific Community, sondern auch von der Organisation ,geschätzt“ zu werden. Sie bekommt zwar nur ein quantifizierendes und kein auf die Projektinhalte bezogenes Feedback. Dennoch nimmt sie es als Entlastung wahr, innerhalb des Bewertungssystems auch als forschende Professorin sichtbar zu werden.

Die Auseinandersetzung mit den universitären Leistungsbewertungen bleibt für Frau Lange jedoch weiterhin virulent. Denn sie sieht sich auf informeller Ebene - ,jedes Mal“, wenn sie „mit einem Dekan oder irgendwem spreche“ mit der organisationalen Erwartung konfrontiert, möglichst bald „eine Forschergruppe oder sogar nen SFB“ mit zu initiieren. Sie lehnt Verbundforschung zwar nicht grundsätzlich für sich ab. Problematisch ist für sie jedoch die Tendenz, solche Forschungsformate von oben zu „dirigieren“. Zum einen sei es „Unsinn“, eine neu berufene Professorin wie sie in die Pflicht zu nehmen, die noch vor der Herausforderung stehe, als Kooperationspartner passende KollegInnen „überhaupt erst mal kennen[zu]lernen“. Zum anderen müssten Forschungskooperationen grundsätzlich von den WissenschaftlerInnen selbst ausgehen, da nur diese einschätzen könnten, ob und inwieweit sich ihre jeweiligen Forschungsinhalte und -paradigmen in produktiver Weise miteinander verbinden ließen. Frau Lange behauptet damit die Eigenlogik wissenschaftlicher Forschung gegenüber der eher managementorientierten Vorstellung, top-down neue Forschungsteams bilden zu können.

Trotz dieser klaren Haltung kann sie sich nur bedingt von den an sie gerichteten Erwartungen abgrenzen. Denn anders als Herr Timme sieht sich Frau Lange als relativ neu berufene W-Professorin unter einem, wie sie sagt, „permanenten 
Druck“. Sie könne sich schon aufgrund dieser Position „,nicht einfach hinsetzen und sagen: ,Mach ich nicht.“ “ Hinzu komme, dass die Konkurrenz um größere Drittmittelerfolge auch das kollegiale Verhältnis in der Fakultät überlagere:

\begin{abstract}
„Aber es ist letztlich so, dass es natürlich sich so stark verselbstständigt hat und intern man dauernd dann vorgeführt bekommt, dass natürlich die mehr wert sind, die eben mit Drittmitteln aufwarten können, dass man das gar nicht so einfach ignorieren kann. Man könnte es vielleicht. Nein, aber es führt dazu, also wir hatten neulich hier so'n Fall, dass da manche Kollegen, ein Kollege hatte sehr viele Anträge gestellt und die wurden dann immer nicht bewilligt und der war so unter Druck. Das führt dann manchmal dazu, dass sozusagen egal welches Thema, egal ob man dazu wirklich, ob da das Herzblut drinhängt oder ob man dazu viel gearbeitet hat, Hauptsache es bietet sich die Gelegenheit. Also dann hat er sozusagen dann ein Thema mit beantragt, was eigentlich mein Thema gewesen wäre so, ne.“
\end{abstract}

Die Fakultät bietet somit keinen Rückzugsraum, in dem man sich auf eine kollegiale Solidarität gegenüber den organisationalen Leistungsbewertungen verlassen könnte. Das Beispiel des Kollegen, der Drittmittel für ein Forschungsthema beantragte, welches eigentlich zu Frau Langes Schwerpunkten gehörte, verweist hier vielmehr auf Entsolidarisierungstendenzen - auch wenn der betreffende Kollege damit selbst auf den zunehmenden „Druck“ reagierte.

Mit Blick auf das kollegiale Verhältnis nimmt Frau Lange auch die Organisation als nur wenig hilfreich war. So würden etwa Informationen zu Projektausschreibungen teilweise nur an ,ausgewählte Fachkollegen“ weitergegeben:

„Also total undemokratisch, ne, und man selber erfährt dann vielleicht erst kurz vor Ablauf der Bewerbungsfrist davon, wo man dann sagt: ,Ja. Hätte ich da eher von gewusst, hätte ich mir vielleicht auch was überlegen können, aber so.““

Frau Lange sieht sich hier mit einer informellen Hierarchie konfrontiert, die sich für sie mit einem Wettbewerbsnachteil verbindet. Als ähnlich hierarchisch und intransparent beschreibt sie die organisationale Informationspolitik in Bezug auf Lehrdeputatsreduktionen. So hätte es am Fachbereich ,gerade einen Fall“" gegeben,

„wo dann jemand aus unerfindlichen Gründen, das ist dann manchmal auch so eine Feudalstruktur, wo das dann auch gar nicht mehr transparent ist, warum jemand jetzt plötzlich viel weniger lehrt als alle anderen. Das ist nicht schön. Also da braucht man schon transparente Strukturen. [...] Und wenn man dann nachfragt, dann heißt es so nach dem Motto: ,Mischen Sie sich doch nicht ein!' Kann man zur Kenntnis nehmen, aber ist natürlich nicht gerade im Sinne eines gedeihlichen Auskommens und einer Transparenz." 
Frau Langes hier noch deutlicher geäußerte Kritik an der internen Kommunikation ist schon deshalb interessant, weil mit der Einführung neuer Formen der Leistungskontrolle und -bewertung im Sinne des NPM eigentlich eine größere Transparenz erreicht werden sollte. In der Beschreibung von Frau Lange verbinden sich die neuen Governance-Strukturen demgegenüber mit „feudalen“ Verteilungsmechanismen. Die Stärkung der hierarchischen Steuerung - sie spricht an anderer Stelle explizit davon, dass ,die Hochschulleitung sich wirklich so versteh[e], dass alles von ganz oben gemanagt werden“ müsse - korrespondiert nicht mit klaren, für alle nachvollziehbaren Begründungen für die Bevorzugung einzelner ProfessorInnen, sondern mit intransparenten Aushandlungs- und Entscheidungsprozessen, von denen FachkollegInnen selbst bei Nachfrage ausgeschlossen bleiben. In ihrer Wahrnehmung gibt es insofern eine Art ,Inner Circle', dem sie nicht oder zumindest noch nicht angehört. Sie kennt zwar nicht die genauen Gründe, weshalb einige ProfessorInnen in der skizzierten Weise - sei es durch ,exklusive' Informationen oder eine Teilbefreiung von ihren Lehrpflichten - profitieren. Für Frau Lange dürfte sich durch diese Erfahrungen aber weiterhin der Druck erhöhen, über bestimmte Forschungsleistungen an Anerkennung zu gewinnen. Gerade auch, weil sie an ihrer aktuellen Universität kaum über soziales Kapital durch gewachsene persönliche Beziehungen verfügt, scheint eine Bewährung über Drittmittel- und Verbundforschung für sie fast alternativlos jedenfalls sofern Frau Lange nicht zu jenen ProfessorInnen gehören möchte, die als weniger ,wert“ gelten.

\section{Fazit}

Für Frau Lange sind neue Governance und universitäre Leistungsbewertungen insgesamt also mit weit größeren Ambivalenzen als für Herrn Timme verbunden. Ein Grund hierfür ist ihre disziplinäre Zugehörigkeit, durch die sich Frau Lange innerhalb der organisationalen Bewertungsordnung benachteiligt sieht, da einige ihrer Forschungsleistungen weder angerechnet noch anerkannt werden. Aus ihrer Sicht werden Unterschiede zwischen den Fächerkulturen zu wenig berücksichtigt und Leistungskriterien - wie etwa Drittmittel oder Artikel in peer-reviewed Journals - auch auf Disziplinen übertragen, in deren Scientific Community diese keine entsprechende Rolle spielen. Gemessen an den Deutungen einiger LiteraturwissenschaftlerInnen, die sich primär, wie wir in Kap. 3 gezeigt haben, als IndividualforscherInnen verstehen und Drittmittel- sowie Verbundprojekte per se ablehnen, lässt sich bei Frau Lange allerdings von keiner unüberbrückbaren Differenz zwischen ihren eigenen fachlichen und den organisationalen Qualitätsmaßstäben sprechen. Sie sieht das große Gewicht der Drittmittel bei den Leistungsbewertungen zwar kritisch. Zugleich konnte sie aber bereits bei der 
LOM von einem ihrer Forschungsprojekte profitieren. Die inneren Konflikte, die für Frau Lange aus den universitären Bewertungsprozessen resultieren, können somit nicht allein mit ihrer disziplinären Zugehörigkeit erklärt werden.

Mindestens ebenso wichtig ist in ihrem Fall die Karrierestufe einer relativ neu berufenen W-Professorin. Frau Lange hat sich aus ihrer Sicht noch keine inneruniversitäre Position erarbeitet, die ihr einen Rückzugsraum gegenüber organisationalen Erwartungen eröffnen könnte. Auch wenn sie fundierte Argumente gegen „von oben“ eingeforderte Verbundformate hat, sieht sie kaum die Option, sich längerfristig zu verweigern. Frau Lange erwähnt zwar an einer Stelle, dass sie eigentlich selbst über die Art und Inhalte ihrer Forschung entscheiden könne, da sie ,ja“ ihre „Stelle“ in keinem Fall „verlieren“ würde - das sei ,ja das Schöne an der Verbeamtung". Zugleich kommt sie aber verschiedentlich auf den Druck zu sprechen, unter dem sie aktuell stehe. In diesem Kontext zeigte sich auch die Bedeutung des kollegialen Verhältnisses, mit dem sich Frau Lange ausführlicher auseinandersetzt, als dies bei Herrn Timme der Fall war. Für sie spiegelt sich auch fakultätsintern eine Wettbewerbs- und Anerkennungsordnung wider, in der eine Forschung jenseits von Drittmitteln und Verbundprojekten zu einem spürbaren Verlust an sozialem und symbolischem Kapital führt.

Der doppelte - organisationale wie kollegiale - Fokus auf bestimmte Forschungsleistungen ist für Frau Lange auch deshalb problematisch, weil die Leistungen in der Lehre im Gegenzug eine nur untergeordnete Rolle spielen. Während Herr Timme zumindest partiell von seinen guten Evaluationsleistungen profitieren konnte, nimmt sie eine deutliche Nicht-Anerkennung ihres hohen, zeitaufwendigen Engagements im Bereich der Lehre und Nachwuchsförderung wahr. Der Anspruch einer Aufwertung dieser professoralen Kernaufgabe wird aus Sicht von Frau Lange an ihrer Universität keineswegs eingelöst. Daran ändert für sie auch die Tatsache nichts, dass jüngere ProfessorInnen wie sie bei ihren Bewerbungen ein Lehrkonzept einreichen mussten. Denn dieser - nur vermeintlichen - Aufwertung der Lehre steht schließlich ihre danach gemachte Erfahrung gegenüber, für ihr Engagement als Lehrende weder mit nennenswerten LOMPunkten belohnt noch auf andere Weise seitens der Organisation anerkannt zu werden. Dass vielmehr drittmittelstarke ProfessorInnen mit einer Teilbefreiung von ihrer Lehrverpflichtung belohnt werden, unterstreicht für Frau Lange nur zusätzlich, dass die Bewährung über, gute' Lehre eindeutig im Schatten bestimmter Forschungsleistungen steht.

Aus einer interessengeleiteten Perspektive wäre hieraus die eindeutige Konsequenz zu ziehen, sich um eine Reduzierung des Lehrdeputates oder zumindest um eine routiniert-pragmatische Erfüllung der Lehrpflichten zugunsten größerer Freiräume für die Forschung zu bemühen. Für Frau Lange bedeutet die 
geringe Wertschätzung der Lehrleistungen jedoch ein Dilemma, das sie nicht einfach - im Sinne einer strategischen Entscheidung - für sich auflösen kann. Denn aufgrund ihres hohen Lehrethos und des „Spaßes“, den sie an der Lehre hat, werden interessengeleitete Erwägungen bei Frau Lange sehr deutlich von identitätsbezogenen Ansprüchen überlagert. Dies zeigte sich eindrücklich gleich zu Beginn des Interviews, als sie davon sprach, trotz des fehlenden ,Pay Offs“ bei der LOM „das Gefühl“ zu haben, Qualifizierungsarbeiten weiterhin ,wirklich lesen und wirklich begutachten" zu müssen.

Eine stärkere Anpassung an die organisationalen Erwartungen und Leistungsbewertungen zulasten der Lehre ist für Frau Lange insofern ein schwieriger, zweischneidiger Schritt. Sie äußert entsprechend, diese „Konsequenz noch nicht richtig gezogen“ zu haben, obwohl sie deutlich „merke“, den zeitlichen Ressourcenkonflikt zwischen Forschung und Lehre eigentlich nur so bewältigen zu können: „Besonders, wenn ich noch mehr Verbundforschung zum Beispiel mir organisieren will, dann brauch ich mehr Zeiträume, also wo muss man kürzen? Das geht dann ja fast nur da." Unabhängig davon, inwieweit Frau Lange tatsächlich ihren zeitlichen Aufwand für die Lehre „kürzen“ wird, verweist ihr Beispiel auf erschwerte Bedingungen für jüngere ProfessorInnen, langfristig einer hohen intrinsischen Lehrmotivation zu folgen. Schließlich laufen sie durch die universitären Leistungsbewertungen Gefahr, zu den „Verlierern“ zu gehören und Nachteile bei der weiteren Karriereplanung in Kauf nehmen zu müssen.

Wir werden solche Spannungsverhältnisse zwischen Forschung und Lehre und deren Folgen für die Deutungen von und den Umgang mit Leistungsbewertungen im nächsten Kapitel noch weitergehend betrachten. Als drittes Beispiel möchten wir nun aber einen Fall darstellen, der sich in verschiedenen Hinsichten von den beiden bis hier geschilderten unterscheidet. Hierbei handelt es sich um einen jüngeren Politikwissenschaftler, dessen Perspektive auf die Hochschulreformen von einer - im Vergleich zu Frau Lange - anders gelagerten und noch tiefergehenden Ambivalenz geprägt ist.

\subsection{Herr Pauls: Leistungsbewertungen, aber richtig! (POL5)}

Der Politikwissenschaftler Herr Pauls wurde nach Stationen im europäischen Ausland vor einigen Jahren auf seine erste W3-Professur an die derzeitige Universität berufen. Er hat kürzlich das Amt des Studiendekans übernommen, dessen Ausübung zum Zeitpunkt des Interviews einen Schwerpunkt seiner Tätigkeit 
darstellt, was seine Perspektive auf die organisationalen Leistungsbewertungen im Gespräch maßgeblich prägt. Herr Pauls äußert sich nicht nur als von universitären Leistungsbewertungen betroffener Hochschullehrer, sondern auch als Inhaber eines Amtes, das der Vermittlung der organisationalen Zielvorstellungen an die KollegInnen dient. ${ }^{9}$

Unabhängig von diesem, für ihn noch neuen, Amt macht Herr Pauls sein Engagement als Lehrender sehr explizit und rückt seine Freude an der Zusammenarbeit mit Studierenden ins Zentrum des Gespräches. Die universitäre Lehre nimmt einen großen Raum im Interview ein, in dessen Verlauf Herr Pauls die Leistungsbewertungsverfahren wiederholt hinsichtlich ihrer Ausgestaltung und Auswirkungen im Bereich der Lehre reflektiert. Die zunehmende Professionalisierung der Organisation und Qualitätssicherung dieses Bereiches wird von ihm genauso wie der derzeitige Aufbau einer universitätseigenen Controllingabteilung mit Interesse verfolgt und nicht im Sinne eines drohenden Managerialismus gedeutet. Angesichts eines von ihm kritisierten organisationalen Fokus auf Forschungsleistungen, verstanden vor allem als Drittmitteleinwerbung, müsse man allerdings auch danach fragen, wo die Lehre in diesem System letztlich „bleib[e]“. Ein besonderes Augenmerk richtet sich in seinem Fall auf die nicht-intendierten Effekte, aber auch Potenziale der neuen Governancestrukturen in der Lehre sowie auf die persönlichen Schlussfolgerungen, die Herr Pauls aus seinen Beobachtungen zieht.

\section{Perspektive auf NPM zwischen Hoffnung und Sorge}

Unmittelbar von den universitären Leistungsbewertungen ist Herr Pauls vor allem aufgrund der Ziel- und Leistungsvereinbarungen betroffen, die er im Rahmen seiner Berufung mit dem Präsidium einging. Neben der Einwerbung einer bestimmten Drittmittelsumme gehören hierzu aktuell drei Publikationen pro Jahr in peer-reviewed Journals und - interessanterweise - verpflichtende Lehrevaluationen, an deren Ergebnissen seine Lehrleistung zu bewerten sei. Letzteres ist deshalb interessant, da die Lehre explizit auf seinen Wunsch hin Bestandteil seiner individuellen Leistungsbeurteilung wurde. Durch diese Verbindlichkeit habe Herr Pauls der hohen Relevanz, die er der Lehre beimisst, Ausdruck verleihen wollen, wie er betont:

\footnotetext{
${ }^{9}$ Es handelt sich im Fall von Herrn Pauls jedoch um keines der ergänzenden Experteninterviews, die wir u. a. mit Studiendekanen geführt haben. Er wurde explizit als betroffener Hochschullehrer und Fachvertreter der Vergleichenden Regierungslehre adressiert.
} 
„Ich bin wahrscheinlich der Einzige in [Name Stadt], in dessen Leistungsvereinbarung drin steht, dass er auch an seinen Lehrevaluationen gemessen werden soll, [...] ich hab das selbst vorgeschlagen. Ich hätte mich teilweise ohrfeigen können in den letzten paar Semestern ((lacht)), weil da schaut man auf die Lehrevaluation und denkt: ,Oh. Fanden doch nicht alle toll, was du gemacht hast, obwohl du dir so viel Mühe gibst.' Aber ich hatte wirklich angestrengt, dass das bei mir drin steht. [...] Weil mir die Lehre so wichtig ist, und danach hab ich mir gedacht teilweise: ,Bist du mit nem Klammerbeutel gepudert? Was hast du da gemacht?““

Die auf Fachbereichs- und Institutsebene bestehenden - kollektiven -Zielvereinbarungen spielten in seinem beruflichen Alltag indes eine untergeordnete Rolle und adressierten sehr allgemeine Kategorien, die zwar ,irgendwie mit Leben gefüllt werden“ müssten, aber ,nicht wirklich [...] quantifiziert“ seien. Auf seine eigene Arbeit wirkten sich die vergleichsweise abstrakten Zielerwartungen daher kaum aus. Durch die LOM sieht er sich ebenfalls keinem konkreten Anpassungsdruck ausgesetzt. Man habe sich auf Institutsebene auf eine gleichförmige Mittelverteilung ,geeinigt", während es an anderen Instituten ,,eher so geregelt [sei], dass dann teilweise auf Antrag da irgendwie Gelder fließen“. Hinsichtlich der Sinnhaftigkeit der LOM ist Herr Pauls indes skeptisch, da sie ,erstens mehr Arbeit" bedeute und die damit erhoffte Effizienz zweitens daher eher fraglich sei.

Eine von den Leistungsbewertungsprozessen ausgehende problematische Steuerungswirkung, wie sie von einigen ProfessorInnen in Beiträgen in F\&L, aber auch von Frau Lange, angesprochen wurde, sieht Herr Pauls also zunächst nicht. Hinzu kommt, und dieser Punkt wird mit Blick auf seinen Umgangsweise mit den an ihn gerichteten universitären Leistungserwartungen noch bedeutsam werden, dass er sich einer organisationalen Zielerwartung nicht ausgeliefert fühlt. Seine Leistungsvereinbarungen beschreibt er mehr als das Ergebnis einer kooperativen Aushandlung denn einer hierarchischen Durchsetzung. Letztlich ginge der „Impuls“ ja ,nicht vom Präsidium [...], sondern von den individuellen Hochschullehrern“ aus, die eben „Möglichkeiten“ hätten, Leistungsstufen zu vereinbaren, sodass er dieses Governance-Instrument als weitestgehend selbstbestimmt erlebt:

„,[M]an weiß ja, wie der Hase läuft, insofern, wenn man hier als Neuberufener ankommt, dann kriegt man ja die Möglichkeit, gleich Leistungsstufen zu vereinbaren. Und von daher weiß man dann auch, man selbst kann auf den Geschäftsführer zugehen und das mit dem besprechen, verhandeln und dann entscheiden, und dann wird nach drei Jahren wieder geguckt."

Da er sowohl über die Inanspruchnahme von Leistungszulagen entscheiden als auch über die Ausgestaltung der Zielvereinbarungen mitbestimmen könne, sieht Herr Pauls sich nicht als jemand, dem Ziele einfach diktiert würden, sondern 
als jemand, der diese aktiv mitgestalten kann. Anders sieht es jedoch bei den Leistungsbewertungskriterien in Sachen Forschung aus. Hier erachtet er die Zielvorgaben als restriktiv. Seine kritische Bewertung wird bereits zu Gesprächsbeginn erkennbar:

„Ja, es gibt einen Wandel, und der Wandel ist, dass Drittmitteleinwerbung immer wichtiger wird auf Kosten aller anderen Bereiche, also sowohl der ganzen Säule Lehre würde ich sagen, als auch anderer Aspekte im Bereich Forschung, wie beispielsweise, wo man publiziert, in welchen Zeitschriften man publiziert, vielleicht sogar auch andere Kennzahlen, unter anderem wie oft man zitiert wird und andere Dinge."

Dass er den beschriebenen „Wandel“ nicht befürwortet, verdeutlicht er schon durch die gewählte Formulierung, dieser werde ,auf Kosten aller anderen Bereiche" vollzogen, was eine übersteigerte Fixierung auf rein monetäre, messbare Faktoren vonseiten der Universität nahelegt. Zugleich klingen zwei Herrn Pauls wichtige, jedoch kaum berücksichtigte Aspekte der Bewertung wissenschaftlicher Leistungen an, die im weiteren Interviewverlauf thematisch sind: erstens die bereits erwähnte Verbesserung der Lehre sowie zweitens die Relevanz von Publikationen als Beurteilungsbasis von Forschungsleistungen. Überhaupt geht es Herrn Pauls um ein differenzierteres Beurteilungssystem anstelle einer selektiven Berücksichtigung von Bewertungsdimensionen, wie er an mehreren Stellen des Interviews betont. Seine mehrjährigen Auslandserfahrungen sind in diesem Zusammenhang nicht unwichtig, da sie ihm einen direkten Vergleich der deutschen Umsetzung von NPM mit ,zentralen Vorreitern“ ermöglichen. ${ }^{10}$

Vor diesem Hintergrund bewertet Herr Pauls die implementierten Leistungsbewertungsverfahren an der eigenen Universität, wie auch hierzulande insgesamt, als unausgereift. Während er den Status quo an den deutschen Universitäten noch eher als „,die Steinzeit des New Public Management“ begreift, erlebte er im Ausland einen durchgreifenden Managerialismus, den er nicht durchweg negativ deutet. Denn obgleich ihm - anders als zuvor - nun erspart bleibe, ,die ganze Zeit irgendwelche nervigen Fragebögen ausfüllen“ zu müssen, beurteilt er den derzeitigen Umsetzungsstand in Deutschland als noch in den Anfängen steckend:

„Anfänge des New Public Managements heißt, wir messen erst mal ganz grob, und wir messen erst mal das, was ganz einfach zu messen ist.“

\footnotetext{
${ }^{10}$ Hierin spiegeln sich die zeitlich sehr unterschiedlich verlaufenen Implementationsprozesse der NPM-Reformen zwischen den Nationen wider, die wir bereits in Kap. 1 angesprochen haben.
} 
Faktisch seien dies die erwähnten Drittmitteleinnahmen, während die Lehre, aber auch schwieriger zu quantifizierende Forschungsleistungen weitgehend ausgeklammert blieben. Konkreter auf seine eigene Person bezogen bedeutet dies eine eindeutige Erwartungshaltung der Hochschulleitung, deren „Masterplan Drittmittel“ für Herrn Pauls eine „Hürde“ darstellt, die weder an seinen eigenen Zielvorstellungen und Forschungspraktiken ausgerichtet noch mit diesen verträglich sei. Für ihn überwiegt dadurch der Eindruck, primär unter einem Drittmitteldruck zu stehen, während andere Tätigkeitsaspekte keine hinreichende Berücksichtigung finden. Es gebe zwar ,die Möglichkeit, Leistungsvereinbarungen einzugehen mit dem Präsidium“, allerdings sei es an seiner Universität

\begin{abstract}
„dann [...] so, dass Forschung und Lehre genannt werden, dass aber, wenn man es ein bisschen genauer liest, man realisiert: Es geht primär um Drittmittel. Und das ist ein bisschen so, wie zum Metzger zu gehen und zu sagen: ,Ich hätte gern so und so viel Pfund Hack. ' Und dann sagt er: ,Das kostet so und so viel.' Ja insofern, als relativ klar vorgegeben ist, für wie viel $1000 €$ Drittmittel man dann wie viel $100 €$ Plus am Ende des Monats rausbekommt. Während im Bereich der Lehre dann da unter anderem steht, man solle doch an der Lehrevaluation teilnehmen und seine Folien ins Internet laden, was auch wichtig ist, aber nicht ganz so schwierig wie Tausende von Euros an Drittmitteln an Land zu holen.“
\end{abstract}

Die beschriebene Fokussierung seiner Universität sei zu Teilen zwar der Suche nach einer praktikablen Form der Beurteilung und mangelnden „Kapazitäten“ für die Umsetzung eines differenzierteren Beurteilungssystems zuzuschreiben, ${ }^{11}$ vor allem aber erkennt der Politikwissenschaftler in dieser Zuspitzung auf einige wenige Parameter eine Hierarchisierung zum Nachteil der Lehre wie auch anderer Aspekte seiner Forschungstätigkeit. Schließlich seien auch „Impact-Faktoren [...] nen hartes, messbares Kriterium“, das „einfach [zu] berechnen“ sei. Herr Pauls habe zwar im Rahmen seiner Ziel- und Leistungsvereinbarungen mit der Hochschulleitung in bestimmten Punkten, so etwa den Publikationsformaten, „relativ frei verhandeln“ können, nicht aber ,bezüglich dieser monetären Geschichten.“

Mit Zitationszahlen, Impact-Faktoren oder Publikationsorganen zeigt der Politikwissenschaftler alternative - durchaus auch quantitative - Beurteilungskriterien auf, die er nicht per se ablehnt. Herrn Pauls geht es nicht schlichtweg

\footnotetext{
${ }^{11}$ Dies sieht er auch durch Äußerungen aus dem Präsidium seiner Universität bestätigt: „Zwei ganz zentrale Spieler bei uns im Präsidium haben zu mir vor ner Weile nur halb im Scherz gesagt: ,Wir schauen uns effektiv nur Drittmittel an, weil man Geld gut zählen kann und messen kann, und alles andere ist schwierig, und deswegen machen wir es im Moment nicht. ““
} 
um eine Kritik an einer Quantifizierung seiner Leistungen, vielmehr sieht er einen Veränderungs- bzw. Verbesserungsbedarf auf der Ebene der Umsetzung der universitären Leistungsbewertungen. Den Rückgriff auf die Bibliometrie bei der Beurteilung seiner Leistungen fände er - richtig eingesetzt - eigentlich sogar ,wunderbar":

„Ich meine, wenn es wirklich um bibliometrische Analysen geht, kann man natürlich auch alles noch ein bisschen ausgefuchster machen, und natürlich kann man sich überlegen, inwiefern man sowohl mit Zitationsindizes arbeitet, als auch mit am Impact-Faktor orientierten Werten. Aber ich denke schon, also im Moment ist es einfach zu einseitig und alles andere, was davon bisschen weg geht, alles was auch nur stückweit in eine andere Richtung geht, ist schon mal besser.“

Herr Pauls lässt in seine Kritik an Leistungsbeurteilungen insofern konstruktive Vorschläge einfließen, die durchaus im Einklang mit der Idee der Überprüfung professoraler Leistungen stehen. Wie wir noch zeigen werden, verbindet Herr Pauls auch hinsichtlich der Lehre seine Kritik am Status quo mit konkreten Vorschlägen, wie der Geringschätzung dieses Bereichs durch eine konsequentere Umsetzung der Leistungsbewertungen entgegengewirkt werden müsse. Hier erkennt er geradezu ein enormes, bei weitem nicht ausgeschöpftes Verbesserungspotenzial.

Bevor wir uns Herrn Pauls diesbezügliche Einschätzungen und seine hieraus gezogenen handlungspraktischen Konsequenzen genauer anschauen, möchten wir jedoch zunächst seine Ansprüche an ,gute" Lehre und Forschung genauer betrachten, die Ausgangspunkt seiner Kritik und Vorschläge darstellen.

\section{Lehre: Orientierung an internationaler Forschung als Maßstab}

Während Herrn Pauls in den ersten Semestern des Bachelor-Studiengangs vor allem an der Vermittlung politikwissenschaftlicher Grundlagen und einer ersten Orientierung im Fach gelegen ist, verbindet er seine Forschungs- und Lehrthemen in den späteren Semestern sehr eng miteinander. Blickt man auf seine Schilderungen zufriedenstellender Lehre, beschreiben diese vor allem die wiederkehrenden Möglichkeiten der Integration von Lehre und Forschung. Anders als Herr Timme etwa, dessen Forschungsthemen in die Lehre eher einseitig einfließen, geht Herr Pauls dabei stärker von einer Wechselseitigkeit aus. So greift er die Gegenstände seiner Seminare oder Vorlesungen auch in der eigenen Forschung auf und publizierte in der Vergangenheit bereits mit Studierenden gemeinsame Artikel. Vor allem ist Herrn Pauls daran gelegen, die Studierenden möglichst früh, möglichst ,niedrigschwellig“, an die politikwissenschaftliche Forschung heranzuführen, womit in seinem Fall eine stark methodische 
Ausrichtung seiner Lehrziele verbunden ist. In seinen Vorlesungen diskutiert er etwa neue, meist empirische Arbeiten, wobei er dann immer

„versuche [...], deutlich zu machen: Worum geht's, was ist der Stand der Forschung bzw. was ist die Literaturbesprechung, die die Autoren da präsentieren, Daten und Methoden, dann Ergebnis und dann Kritik? Und das ist mir wichtig, weil das einfach die zentralen Basics in dem Forschungsdesign sind und man sich daran eigentlich schön abarbeiten kann [...]. Darum geht es mir effektiv. Ja um Forschungsdesigns, um angewandte Forschungsdesigns, und da versuche ich die Leute eigentlich so schnell daran zu führen, wie es geht, weil ich denk, dass es wichtig ist, weil ich denk, dass es in Teilen viel zu wenig auch gemacht wird.“

Diese ihm wichtigen möglichst aktuellen und originären - und insofern nicht didaktisch aufbereiteten - Einblicke in den internationalen Forschungs- und Diskussionsstand auf seinem Gebiet erfolgten anhand zumeist englischsprachiger Zeitschriftenartikel. Auf diese sehr ,praxisorientierte“ Weise würden die Studierenden an die politikwissenschaftliche Forschung „herangeführt“, die diese im Idealfall in kleinerem Umfang ,relativ schnell replizieren“ könnten, wodurch sie auch selbst in die Lage versetzt würden, zu den „Lösungen“ gesellschaftlicher Problemlagen durch die Beantwortung dringlicher Fragen beizutragen. ${ }^{12}$

Den ihm wichtigen möglichst frühen Forschungsbezug der Lehre fördert Herr Pauls nicht nur durch seine Lehrpraxis, sondern ebenso durch die Einbindung der Studierenden in die eigenen Forschungskontexte. Er beschreibt diese Form der Integration von Lehre und Forschung als einen eher spontanen bzw. naturwüchsigen denn geplanten Prozess. Die Publikationen mit Studierenden hätten sich aus einer gemeinsamen Beschäftigung mit dem Forschungs- und Lehrgegenstand heraus ,eher [...] so ergeben“. Er bewertet dies im Nachhinein als eine „natürlich schöne Sache“, die motivierend sei, sodass er den Studierenden seiner aktuellen Veranstaltungen gerne von solchen „Highlights“ bzw. „Leuchttürmen“ berichte:

„,[D]ie haben einfach ihre Sachen in Teilen dann gemeinsam mit mir publiziert und haben jetzt sehr spannende Karrieren [...]. Das sind einfach Leute, die ein Interesse daran entwickelt haben und dann ein Stück des Weges mit mir auch mitgegangen

\footnotetext{
${ }^{12}$ Dies wird von Herrn Pauls nicht zuletzt mit der aus seiner Sicht weniger voraussetzungsvollen Forschungspraxis der Sozialwissenschaften begründet, die anders als Disziplinen wie etwa die „Medizin oder Biologie“ zumeist keinen hochgradig kodifizierten Stand der Forschung (Gläser et al. 2010) aufweisen, der eine frühe Einbindung Studierender in Forschungskontexte erschwert.
} 
sind. Bis sie halt ihre Bachelorarbeit dann vielleicht auch nur in Anführungszeichen fertig hatten. Das erwähne ich dann auch ganz gerne, also was man damit alles machen kann."

Sein eigenes Anspruchsniveau in der Lehre beschreibt er aufgrund seiner internationalen und methodisch-statistischen Ausrichtung als vergleichsweise hoch. Er sieht sich selbst dabei aber zugleich in einem Lernprozess, da er zwar um eine möglichst gewinnbringende Erfahrung auf beiden Seiten bemüht sei, seine Lehrziele und Anforderungen von den Studierenden jedoch nicht immer verstanden würden und diese teils verunsicherten. Umso wichtiger sei ihm ein entsprechendes Feedback der Studierenden, um die Qualität seiner Lehre kontinuierlich zu verbessern. Umgekehrt will er die Studierenden

„wirklich, so gut wie es geht in diesem Setting, an die Hand nehmen und auch versuchen, ihnen die Angst zu nehmen und ihre Neugierde zu wecken. Und in Teilen klappt es, in Teilen klappt es aber auch nicht, und das ist für mich noch immer so das große Rätsel: ,Wie kann ich es besser machen?`Weil: In Teilen klappt es nicht. [...] Und ich reiß mir nen Arm aus und noch einen und ein Bein, wenn es sein muss auch noch, und es klappt trotzdem nicht, hab ich den Eindruck."

Hier zeigt sich eine Parallele zu Frau Lange. Anders als Herr Timme begreifen beide Lehrende das Instrument Lehrevaluation nicht primär als eine Sichtbarmachung möglichst positiver Evaluationsergebnisse, sondern vorrangig als eine wichtige Hilfestellung, die als Kommunikationsmedium zwischen Studierenden und Lehrenden fungiert. Somit verwundert es auch nicht, dass Herr Pauls dieses Instrument - trotz methodischer Einwände - nutzt. Hierauf werden wir bei seiner Perspektive auf die Möglichkeiten der Leistungsmessung und -verbesserung noch genauer eingehen.

\section{Forschung: Rückzug aus der ,Jagdgemeinschaft“}

Wendet man sich nun der Forschung zu, sieht sich Herr Pauls vor allem angesichts der derzeitigen Governance und ihrer lokalen Umsetzung mit konkreten Problemen konfrontiert, da die organisationale Bewertungs- und Anerkennungsordnung solche ForscherInnen in eine marginale Position versetze, die - so wie er - lieber ihr „eigenes Ding“ machten.

Als problematisch erachtet Herr Pauls neben der fehlenden Berücksichtigung der Lehre die in der Forschung sich abzeichnenden Folgen einer einseitigen Bewertungsordnung. Damit steht seine Einschätzung Frau Langes Deutung eines subalternen Ranges der Lehre und der lehrorientierten gegenüber drittmittelstarken KollegInnen nahe. Er berichtet allerdings weniger von mit der lokalen 
Reformumsetzung verbundenen kollegialen Spannungsverhältnissen, sondern kritisiert zunächst die aktuelle Förderpolitik an sich. So beobachte er eine ZweckMittel-Verkehrung in kooperativen Forschungsinitiativen, die zunehmend von der Idee der Drittmitteleinwerbung und weniger von inhaltlichen Aspekten bestimmt seien:

„Es werden ja einfach immer mehr Initiativen, dass man immer mehr, das ist son bisschen, ich weiß gar nicht ob Reise nach Jerusalem die richtige Metapher ist, ja aber es [...] werden immer mehr Jagdgemeinschaften gegründet, und ich hab da auch mitgemacht. Mache auch in Teilen noch nen bisschen mit [...]. Und ich hab mich da auch zurückgezogen in Teilen, weil es mir einfach zu viel wird, aber auch, weil ich in Teilen der Meinung bin, dass das dort schon einfach viel zu drittmittelorientiert ist.“

Für Herrn Pauls ist zufriedenstellende Forschung vor allem ein weitestgehend offengehaltener, zunächst mehr spielerischer denn systematischer Prozess, der außerhalb konkreter Projektkontexte bzw. Forschungsverbünde angesiedelt ist. Entsprechend bedürfe es keines finanziell oder zeitlich abgesteckten Rahmens, womit er sich von einer überbordenden „Projektförmigkeit der Forschung“ (Torka 2009) distanziert. ${ }^{13}$ So expliziert er:

„Ich bin sowieso einer, der die Sachen nicht ganz so gerne - in Anführungszeichen und vielleicht auch nicht so gut planen kann, wie das bei einigen Kollegen der Fall ist [...]. Also ich versuche auch, das Spiel son bisschen mitzuspielen, wobei ich finde es eigentlich schöner, sich einfach erst mal hinzusetzen mit irgendwelchen Daten.“

Da letzteres bei ihm zumeist anhand von „Sekundärdaten“ geschehe, seien nicht detaillierte Erhebungs- oder Projektpläne gefordert, sondern primär Zeit und Möglichkeiten dafür, sich „einfach erst mal hinzusetzen“ und Berechnungen anzustellen, deren Potenzial für weitergehende Forschungen zunächst offen bleibe - oder wie er es ausdrückt: „Sachen zusammenschmeißen, rödeln und gucken, was bei rumkommt“. Herr Pauls versteht sich folglich eher als ein Individualforscher, der die ergebnisoffene Durchführung - alleine oder mit seinem Lehrstuhlteam - kleinerer Untersuchungen bevorzugt, die anfänglich

\footnotetext{
${ }^{13}$ Entsprechend merkt er an: ,Also die Frage ist ja erst mal: Was ist überhaupt ein Projekt, könnte man sagen? Also ist ein Projekt nur, was irgendwie gefördert ist finanziell mit einer gewissen Summe? Also wenn es das ist, ist es bei mir extrem wenig. " Noch grundsätzlicher hinterfragen Niklas Luhmann (1990, S. 336-340) und Rudolf Stichweh (1994, S. 164-168) projektförmige Forschung.
} 
oftmals mehr lockere Ideen darstellten und sich später möglicherweise zu einer Publikation entwickelten, ohne ,eine Riesensache daraus [zu] machen“. Dies meine eben auch, „nicht großartig“ im Rahmen größerer Verbunde oder Projekte mit anderen WissenschaftlerInnen zu kooperieren:

\begin{abstract}
„Also ich mache gerne mal Sachen auch mit Kollegen zusammen, aber tatsächlich eher im Bereich der universitären Selbstverwaltung als in der Forschung. In der Forschung habe ich es eigentlich am liebsten, mein eigenes Ding zu machen, und wenn ich zusammenarbeite, dann eher mit Nachwuchswissenschaftlern was zu machen. Also da bin ich, glaub ich, auch ganz gut drin so.“
\end{abstract}

Seine bereits im Kontext der Lehre betonte Kooperationsbereitschaft auf „niedrigerem Level“" greift Herr Pauls an dieser Stelle erneut auf, während andere Formen der Forschungskooperation eher abgelehnt werden. Er ist sich darüber bewusst, dass seine Ansprüche an die Forschung nur in geringem Maße mit den Erwartungen der Hochschulleitung übereinstimmen. Für ihn verbinde sich die eigene Forschungspraxis daher oftmals mit dem Eingehen von Kompromisslösungen. Dieses Arrangieren beschreibt er dann wie folgt:

\begin{abstract}
„,[D]agegen kann ich ja nichts tun. Das ist ja ne systemische Veränderung, aber gut, ich mein, ich arrangiere mich so gut damit, wie es geht, also ich mache ja nen bisschen auch was in diesem Bereich. [...] Ich habe ja jetzt gerade nen DFG-Antrag eingereicht und bin auch dabei, nen anderen jetzt zu schreiben, aber das sind halt Einzelanträge und keine Verbundgeschichten. [...] Ich spiele es ein Stück weit, aber nur so wie es für mich noch in Ordnung ist, ohne mich da zu sehr zu zerreißen, weil es halt einfach nicht meine Art des Arbeitens ist."
\end{abstract}

Er legitimiert seine Kompromisse zwar, indem er seine Kontrolle hierüber betont, dennoch sieht er hierin letztlich eine alternativlose Anpassung. Den Konflikt zwischen organisationalem Anspruch und eigener Praxis kann er insofern nur bedingt im Sinne seines Forschungsethos lösen. Aufschlussreich in diesem Zusammenhang ist seine retrospektive Deutung seiner damaligen Entscheidung, das Amt des Studiendekans zu übernehmen. Er stellt diese Entscheidung unmittelbar in den Kontext seiner Kritik an den veränderten Forschungsbedingungen und deutet sie nicht zuletzt auch als Konsequenz eines Konfliktes zwischen subjektiven Ansprüchen und organisationalen Erwartungen:

„,[E]s [ist] in der Tat auch so, dass ich halt auch nen ganz großer Verfechter innerhalb der Uni bin für die Relevanz der Lehre, und vielleicht war das auch dann effektiv einer der Gründe [...], dass ich dann gesagt habe: ,Okay. Dann mache ich halt Studiendekan. “ 
Als „effektive Gründe“ für die Übernahme des Amtes führt er schließlich nicht nur sein persönliches Ziel einer Aufwertung der Lehrtätigkeit an, sondern auch die für ihn „mit Nachteilen“ verbundene ,systemische Veränderung“, mit der er sich „,arrangieren“ müsse. Indes möchte Herr Pauls sein neues Amt voller Tatendrang ausfüllen und dort ,,jetzt erst mal [...] was aufbauen“, also im „Uni-Management“ seine „Nische“ suchen, die er ,ganz spannend finde“ und die er - aus jetziger Sicht bewertet - nach Ende seiner ersten Amtszeit gerne auch verlängern würde:

„Das ist immer für zwei Jahre gewählt, aber wenn es passt, kann ich mir auch vorstellen, das weiter zu machen. Also wir haben da auch wirklich Top-Leute im Dekanat. Bessere Leute kann man nicht bekommen, glaub ich, als die, die wir da haben.“

\title{
NPM, aber richtig! Sympathie und Engagement trotz Kritik
}

Als Studiendekan zeigt sich Herr Pauls geradezu als Befürworter einer konsequenten Leistungskontrolle und Umsetzung von Leistungszielen, die notfalls auch auf hierarchischem Wege erfolgen müssten. Erkennbar geprägt durch seine Auslandserfahrungen wägt der Politikwissenschaftler dabei die möglichen Vor- und Nachteile einer stärkeren organisationalen Einflussnahme ab. Die kaum formalisierten organisationalen Leistungsvorgaben in der Lehre bewertet Herr Pauls zunächst skeptisch, und er ironisiert die bisherigen Bestrebungen eher, als dass er sie als positive Anzeichen eines Strebens nach einem verbessertes Standing der Lehre begreift. So kommentiert er die Handreichungen seiner Universität für bessere Lehre:

\begin{abstract}
„Da steht halt drin: ,Seid nett zu Ausländern. Wirklich! Ja. Also ist drastisch formuliert, aber es geht darum, im Bereich ERASMUS irgendwie Leute zu unterstützen. [...] Und dann steht drin: ,Und publiziert bitte eure Power Point Folien im Internet'. Was nun wirklich keine große Leistung ist. Aber das zentrale ist: Auf der einen Seite haben wir New Public Management und diese Leistungsgeschichten, die aber nur monetär effektiv ausgerichtet sind, und das andere ist natürlich trotzdem die Tatsache, dass deutsche Professoren extrem viel Freiheit haben und eigentlich keinen Vorgesetzten haben. In England und in Holland haben auch Professoren ein Beurteilungsgespräch einmal im Jahr.“
\end{abstract}

Da Lehre hierzulande im direkten Vergleich einen besonders niedrigen Stellenwert habe, gar „,am unwichtigsten“ sei, seien eine höhere Verbindlichkeit und Überprüfung der Lehrleistungen wünschenswert. Die vergleichsweise großen Autonomiespielräume deutscher ProfessorInnen begreift Herr Pauls in diesem Kontext durchaus als nachteilig und spricht von letzteren als ,kleinen Königen“, die ,,in Teilen [...] nicht den Sinn des Ganzen“ sähen. Er plädiert damit zwar nicht direkt 
für eine Beschränkung der ProfessorInnenautonomie. Mit seinem Verweis auf die Praxis anderswo deutet er aber zumindest an, dass verbindlichere Maßnahmen im Lehrkontext wichtig wären. Schließlich habe man ,einfach in Deutschland die Situation, dass Profs eigentlich weiterhin machen können, was sie wollen, trotz New Public Management“. Konkret für die W-Besoldung ausgeführt:

„,[Es gibt ja dieses Grundgehalt und dann halt diese Leistungsvereinbarung. und da, also da bin ich gespannt zu sehen, inwiefern überhaupt an anderen Unis Lehre da schon drin ist.“

Auch wenn er sich persönlich dazu entscheiden könne, seine Lehre ausgehend von den heterogenen Studierendenbedürfnissen didaktisch aufzubereiten, neue Lehrkonzepte auszutesten und deren Erfolg durch die Studierendenurteile überprüfen zu lassen, sieht er auf kollektiver Ebene von den aktuell angewandten Formen der Leistungsbeurteilungen kaum Impulse ausgehen. Schließlich kennzeichne die derzeitige organisationale Beurteilungspraxis vielmehr ein Fehlen eindeutig definierter Beurteilungskriterien und eine ausbleibende Sanktionierung schlechter Lehrleistungen. Er erkennt hier eine organisationale Verantwortung, der die eigene Universität nicht hinreichend nachkomme:

„Es kräht kein Hahn danach, inwiefern es beispielsweise nen großen Unterschied gibt zwischen der Anzahl von Leuten, die sich zuerst mal eingeschrieben haben für ein bestimmtes Modul, und wie viele dann am Ende noch mitmachen. Da gibt es auch große Unterschiede - und die haben wahrscheinlich auch damit zu tun, was gemacht wird im Modul, auch natürlich, was die Prüfungsleistung ist, aber wahrscheinlich auch, wie das didaktisch rüber gebracht wird.“

Gerade auch quantitative Indikatoren dienten der Schwachstellenidentifikation in der Lehre, was deren kontinuierlicher Verbesserung den Weg ebne. Eine ähnliche Aufgabe kommt aus Herrn Pauls Sicht der Lehrevaluation zu, wenngleich diese „mit extrem viel Vorsicht zu genießen“ sei. Dies wird von ihm allerdings methodisch begründet. Das Instrument Lehrevaluation an sich wird nicht zur Diskussion gestellt und sei in jedem Fall „besser als nichts“:

„Also klar hat man Probleme mit der Rücklaufquote. Wir lesen normalerweise im Standardmethodenlehrbuch, wenn die gebiased ist und nicht mindestens so und so hoch ist, dann können wir sie in die Tonne treten. Ich denke mir aber, besser wird es nicht. Das ist das einzige, was wir haben, und was wir von daher wahrscheinlich machen sollten ist, uns das zwar anschauen, aber schon auch uns überlegen, wie das zu den qualitativen Sachen passt, die die Studis geschrieben haben, und wie das aber auch zu anderen Sachen passt.“ 
Da er grundsätzlich die Aussagekraft der verfügbaren Ergebnisse und Kennzahlen nicht anzweifelt, plädiert Herr Pauls in seiner Rolle als Studiendekan folglich auch für eine konsequentere Offenlegung der Ergebnisse der Lehrevaluation:

„Die Ergebnisse der Lehrevaluation werden nicht automatisch an mich weitergeleitet, wenn die Leute das nicht wollen, was ich auch erstmal extrem spannend, aber eigentlich problematisch finde. Ich sag ja nicht, dass die Leute gleich gefeuert werden sollen, wenn sie nicht mindestens eine 3 erreichen in der Lehre. Aber die Information muss an die Leitungsebenen gehen, also sonst können wir das wirklich vergessen. Denke ich.“

Für Herrn Pauls steht damit fest, dass die Lehrevaluation als ein nur individuelles Feedbackinstrument keine zufriedenstellende Handlungswirksamkeit entfaltet. Im Umkehrschluss -wenn nur die verpflichtende Offenlegung Verbesserungen bewirken könne - werden damit von ihm durchaus Zweifel an einer hinreichenden Lehrmotivation der ProfessorInnen nahegelegt. Während er derzeit kaum Möglichkeiten der formellen Steuerung erkenne, habe er dies während seiner Tätigkeit im Ausland anders, und durchaus positiv, erlebt:

\begin{abstract}
„Beispielsweise in [Stadt] gab es Peer Review. Die Senior Leute haben sich tatsächlich rein gesetzt bei den anderen. Und ich glaub schon, dass das eigentlich gut ist. Es ist ja weiterhin so, dass man in Deutschland sowieso Professor wird, ohne irgendeine Lehrbefugnis oder was auch immer zu haben. Die kriegt man ja über die Publikation effektiv [...]. Ich war jetzt schon in relativ vielen Berufungsverfahren, und da war es schon jetzt immer so, dass die Leute nicht nur einen wissenschaftlichen Vortrag halten sollten, sondern auch eine Lehrsimulation. Aber da geht es ja um die Frage: Wer kommt rein? Aber wer drin ist im System, da ist natürlich dann auch die Frage: Wie gut machen die das? Und dann natürlich auch irgendwann vielleicht die Frage: Wie können wir schauen, dass es einfach besser wird?“
\end{abstract}

Dies wird noch weiter ausgeführt:
„Also ich denke, Peer Review ist gut. Ja. Also ich bin da neulich mal über so einen Report vom Wissenschaftsrat von 2008 gestolpert, wo die sagen, wir müssen in Deutschland die Lehrevaluation ernster nehmen und man müsste eigentlich mehr Peer Reviews durchführen und natürlich auch Beurteilungsgespräche. Ist ja auch okay. Aber [...] das macht ja einen Unterschied, ob ich Ihnen erzähle, was ich so mache im Unterricht, im Vergleich dazu, wenn Sie sich mal rein setzten.“

Ein Peer Review in der Lehre, verpflichtende Lehrevaluationen oder Beurteilungsgespräche werden von Herrn Pauls neben der verstärkten Berücksichtigung solcher quantitativer Kennzahlen wie Dropout-Quoten, Prüfungsnoten 
oder Betreuungsrelationen als beispielhafte Maßnahmen der Lehrverbesserung angeführt. Da er ,,kein Gegner der Idee [sei], dass Leute irgendwas leisten sollten in ihrem Job, und dass man versuchen sollte zu schauen, wie man das am besten macht", befürwortet er grundsätzlich die Idee von Leistungsüberprüfungen und deutet diese nicht als illegitime Einschränkung professoraler Privilegien. Eine gewisse Ambivalenz bleibt aber schon aufgrund von befürchteten und in anderen Berufsfeldern beobachtbaren problematischen Konsequenzen, sodass man ,,aufpassen“" müsse, ,die Leute dabei nicht [zu] über[fahren]":

„Ich kann mir wenige Organisationen oder Situationen vorstellen, wo es schwieriger ist, das zu messen, als gerade im Bereich der Wissenschaft. Also das ist auch in anderen Bereichen nicht ganz einfach. Im Gesundheitssektor ist es auch nicht so ganz einfach. Das ist natülich so und das sind ja auch teilweise negative Folgen des New Public Managements, dass man halt nur noch Kennzahlen bedient und bestimmte wichtige Sachen in den Hintergrund dann einfach geraten.“

Der Politikwissenschaftler misst hier - ihm durchaus bewusst - in gewisser Weise mit zweierlei Maß: Einerseits warnt er vor einer Zweck-Mittel-Verkehrung und primären Orientierung an Kennzahlen vor inhaltlichen Kriterien in Forschung und Lehre. Andererseits plädiert er zugleich in der Lehre für eine handlungswirksamere Umsetzung eines Leistungserfassungs- und -bewertungsverfahrens, das derzeit kaum ernst genug genommen werde. Infolgedessen unternehme er als Studiendekan entsprechende erste Schritte, indem er das Spektrum an Möglichkeiten einer Governance für die Lehre auslote, um Maßnahmen für die eigene Fakultät in die Wege zu leiten:

,,[V]ielleicht sind andere Unis da weiter, ich kenne mich da überhaupt nicht aus. Das habe ich mir aber auch auf die Fahnen geschrieben. Also ich hab jetzt jemanden eingestellt, einen ehemaligen Studenten von uns [...], Und ich hab den gebeten, erst mal bisschen auch über den Tellerrand zu gucken und zu schauen, was in anderen Unis in [Bundesland] oder hier in [Region] überhaupt gemacht wird.“

Sein persönlicher Fokus auf die Lehre und seine Hoffnung auf ihre Qualitätsverbesserung, die er mit den Hochschulreformen und der Implementierung von NPM verbindet, lassen den Politikwissenschaftler somit trotz allem zuversichtlich in die Zukunft blicken. Er sieht den Status quo als unbequeme Zwischenphase, während zukünftig vor allem wichtig werde, ein differenzierteres Leistungsbewertungssystem zu implementieren. Dass allmählich ein sich bereits abzeichnender 
Bedeutungsgewinn der Lehre eintreten werde, leitet er allein schon aus deren verstärkter medialer Diskussion wie auch aus neuen Förderinitiativen ab:

„Ich denke, es wird stärker in Richtung Kennzahlen gehen. [...] Ich glaub, die Lehre wird irgendwann später dazukommen [...]. Aber ich denke mir irgendwie auch, wenn man jetzt so die Zeitung liest: Das wird kommen einfach, Qualität der Lehre, das wird kommen. Es gibt ja schon tausende Initiativen und vom BMBF und alles Mögliche. [...] Es kommt. Ja es kommt. Also von daher wird es hoffentlich dann besser $[\ldots] . “$

Für sich selbst formuliert er diesbezüglich ein klares Ziel:

\begin{abstract}
„Wir unterhalten uns ja auch dauernd mit Kollegen mittags dann über unsere lieben Studierenden und was mal wieder nicht geklappt hat und so weiter. Ja. Oder klagt dem anderen auch mal sein Leid oder so [...]. Und dann auch die Frage: Was ist die Erklärung? Wie kann man es besser machen? Und ich bin da ganz gespannt. Also ich hab noch bis [Jahr], bevor ich in den Ruhestand gehe. Also bis dann. [...] Ja ich schraube aber dran. Ich versuche, es besser zu machen. Kontinuierlich.“
\end{abstract}

Die Übernahme des Amtes des Studiendekans, dem er sich, wie er mehrfach betont, nun verstärkt widmen wolle, lässt sich zwar allein schon aufgrund seiner weiterhin betriebenen Drittmittelakquise nicht als gänzlicher Rückzug aus der Forschung interpretieren. Wohl aber sind ein stückweites Zurückweichen aus einem Bereich, dessen Anforderungen seinen persönlichen Ansprüchen zuwiderlaufen, und eine intensivere Fokussierung eines Bereiches zu beobachten, in dem er seine Ansprüche auch deshalb weitgehend realisieren kann, weil er Möglichkeiten des Agenda-Setting erkennt. Während Herr Pauls die entscheidenden Kriterien in der Forschung als „nicht verhandelbare Hürden“ erlebt, bietet sich mit der Lehre ein Handlungsfeld an, in dem er sich aktiv einbringen kann.

\title{
Fazit
}

Gerade weil in den Hochschulreformen auch das Potenzial für eine Verbesserung der Lehrqualität gesehen wird, werden diese von Herrn Pauls nicht grundsätzlich abgelehnt. Erfahrungen in Ländern, in denen NPM weiter fortgeschritten ist, bilden hier Referenzen dafür, was in Deutschland noch werden kann.

Wie deutlich geworden ist, verbinden sich für den Politikwissenschaftler generell gleichermaßen positive wie negative Veränderungen mit implementierten Reforminstrumenten, die ihn sowohl hoffnungsvoll als auch skeptisch stimmen. Diese Ambivalenz ist ihm sehr bewusst und wird im Interview mehrfach 
thematisiert. Es liegt hier also durchaus nahe, dass es nicht zuletzt von seinem Erfolg als Studiendekan abhängig sein wird, inwieweit Herr Pauls zukünftig zu den Reformbefürwortern, gar -promotoren, oder aber den enttäuschten Blockierern gehören könnte. Ob er Nutzen aus den Reformen wird ziehen können oder aber sein Engagement keine Früchte tragen wird, scheint von vielen - noch nicht abschätzbaren - Faktoren abhängig zu sein. Der engagierte Hochschullehrer könnte also noch zum aktiven Blockierer werden oder passiv-resigniert den Rückzug in einen ,Dienst nach Vorschrift' wählen.

\subsection{Subjektive Verarbeitung von Leistungsbewertungen: Erste Anhaltspunkte für Erklärungsfaktoren}

Doch nicht nur Herr Pauls, auch die beiden anderen ausgewählten Fälle zeigen, dass man von keiner klaren Einteilung in GegnerInnen und BefürworterInnen universitärer Leistungsbewertungen ausgehen kann. Keiner der drei Fälle ist entweder durchgängig Gegner oder durchgängig Befürworter der Reformen; und bei allen dreien könnten ihre geäußerten Einschätzungen auch wieder in Fluss geraten, so wie sie ja auch über zurückliegende Einschätzungsveränderungen berichten. Damit verdeutlichen die drei Fälle insgesamt ein sehr differenziertes Verhältnis zwischen professoralen Selbstansprüchen und universitären Bewertungsprozessen.

Fragt man, welche Faktoren bei der subjektiven Verarbeitung von neuen Governance- und Bewertungsformen eine wichtige Rolle spielen können, lassen sich den Fällen erste Anhaltspunkte entnehmen. Wie vermutet, erweisen sich die disziplinäre Zugehörigkeit und die Karrierestufe als erklärungskräftig, um die Haltung zu universitären Leistungsbewertungen zu erklären. So bestätigte sich etwa, dass mit Leistungskriterien wie Drittmitteln in ingenieurwissenschaftlichen Disziplinen eine stärkere Anerkennung der ohnehin vorhandenen professoralen Ansprüche stattfindet, als dies in den Geistes- und Sozialwissenschaften der Fall ist. Ebenso spiegelte sich ein für neu berufene ProfessorInnen größerer Bewährungsdruck in Bezug auf universitäre Erwartungen und Bewertungsmaßstäbe wider.

Zugleich bestätigten sich aber auch die bereits in Kap. 3 herausgearbeiteten innerdisziplinären Unterschiede - wenn etwa GeisteswissenschaftlerInnen nicht per se eine eher ablehnende Haltung gegenüber den organisationalen Leistungskriterien einnehmen. Frau Lange gehört schließlich zu jenen ProfessorInnen 
ihrer Disziplin, die Drittmittelprojekte durchaus in ihre Vorstellungen ,guter ${ }^{\text {* }}$ Forschung integrieren und zumindest partiell von Instrumenten wie der LOM profitieren können und wollen. Um ihre dennoch kritische Sicht auf die universitären Leistungsbewertungen $\mathrm{zu}$ begründen, müssen insofern weitere Erklärungsfaktoren herangezogen werden.

Hier ist zunächst darauf hinzuweisen, dass Leistungsbewertungen in den Bereichen Forschung und Lehre durchaus verschieden wahrgenommen und verarbeitet werden können. Wir können also von keiner beide Tätigkeitsbereiche übergreifenden Ablehnung oder Befürwortung organisationaler Bewertungspraktiken ausgehen. Dies hat insbesondere das letzte Beispiel von Herrn Pauls verdeutlicht, in dessen Fall sich Reformbefürwortung in Sachen Lehre auf der einen mit einem eher skeptischen Beharren auf der eigenen Autonomie in Sachen Forschung auf der anderen Seite kombinierten. Die Ambivalenz der Reformprozesse und die prinzipielle Offenheit des weiteren Geschehens kommen hier besonders deutlich zum Ausdruck.

In den drei Fällen wurde darüber hinaus auch die mögliche Bedeutung lokaler, organisationsspezifischer Faktoren für die subjektive Haltung zu den Reformen sichtbar. Hierzu zählt zum einen die lokale Umsetzung von Leistungsbewertungen. So spiegelten sich in den Interviews etwa Unterschiede hinsichtlich des Verpflichtungsgrades von Lehrevaluationen wie auch hinsichtlich der wahrgenommenen Transparenz organisationaler Kommunikations- und Entscheidungsprozesse wider. ${ }^{14}$ Weiterhin wurde deutlich, dass bei der Verarbeitung universitärer Leistungsbewertungen auch das kollegiale Verhältnis im sozialen Setting des Fachbereichs eine entlastend-kompensierende oder belastende Rolle spielen kann. Bei Frau Lange zeigte sich etwa eindrücklich, dass der Eindruck einer Entsolidarisierung unter den FachkollegInnen die Wirkungsmacht der Leistungsbewertungen noch weiter verstärkt.

Neben der Disziplin und Karrierestufe werden wir auch diese weiteren Erklärungsfaktoren in die empirische Analyse im folgenden Kapitel einbeziehen, in dem wir die professoralen Deutungen und Handlungsstrategien anhand verdichteter analytischer Typen darstellen.

\footnotetext{
${ }^{14}$ Diese ersten Eindrücke passen zu Forschungsergebnisse von Jörg Bogumil et al. (2013), in deren Vergleich verschiedener Universitäten sowohl stärker hierarchische als auch partizipativere Implementationsstile rekonstruiert werden konnten.
} 


\section{Literatur}

Bogumil, Jörg, Martin Burgi, Rolf G. Heinze, Sascha Gerber, Ilse-Dore Gräf, Linda Jochheim, Maren Schickentanz, und Manfred Wannöffel. 2013. Modernisierung der Universitäten. Umsetzungsstand und Wirkungen neuer Steuerungsinstrumente. Berlin: edition sigma.

Bröckling, Ulrich. 2007. Das unternehmerische Selbst. Frankfurt/M.: Suhrkamp.

Bundesbesoldungsgesetz: Bundesbesoldungsgesetz in der Fassung der Bekanntmachung vom 19. Juni 2009 (BGBl. I S. 1434), das zuletzt durch Artikel 2 des Gesetzes vom 9. Dezember 2019 (BGBl. I S. 2053) geändert worden ist, \$35 BBesG Forschungs- und Lehrzulage. http://www.gesetze-im-internet.de/bbesg/BJNR011740975.html.

Gläser, Jochen, Stefan Lange, Grit Laudel, und Uwe Schimank. 2010. The limits of universality: How field specific epistemic conditions affect authority relations and their consequences. In Reconfiguring knowledge production. Changing authority relationships in the sciences and their consequences for intellectual innovation, Hrsg. Richard Whitley, Jochen Gläser, und Lars Engwall, 291-324. Oxford: Oxford University Press.

Heintz, Bettina. 2010. Numerische Differenz Überlegungen zu einer Soziologie des (quantitativen) Vergleichs. Zeitschrift Für Soziologie 39 (3): 162-181.

Liebeskind, Uta. 2011. Universitäre Lehre - Deutungsmuster von ProfessorInnen im deutsch-französischen Vergleich. Konstanz: UVK.

Lohr, Karin, Thorsten Peetz, und Romy Hilbrich. 2013a. Bildungsarbeit im Umbruch. Zur Ökonomisierung von Arbeit und Organisation in Schulen, Universitäten und in der Weiterbildung. Berlin: sigma.

Luhmann, Niklas. 1990. Die Wissenschaft der Gesellschaft. Frankfurt/M.: Suhrkamp.

Stichweh, Rudolf. 1994. Wissenschaft, Universität, Professionen. Soziologische Analysen. Frankfurt/M.: Suhrkamp.

Torka, Marc. 2009. Die Projektförmigkeit der Forschung. Baden-Baden: Nomos. 
Open Access Dieses Kapitel wird unter der Creative Commons Namensnennung 4.0 International Lizenz (http://creativecommons.org/licenses/by/4.0/deed.de) veröffentlicht, welche die Nutzung, Vervielfältigung, Bearbeitung, Verbreitung und Wiedergabe in jeglichem Medium und Format erlaubt, sofern Sie den/die ursprünglichen Autor(en) und die Quelle ordnungsgemäß nennen, einen Link zur Creative Commons Lizenz beifügen und angeben, ob Änderungen vorgenommen wurden.

Die in diesem Kapitel enthaltenen Bilder und sonstiges Drittmaterial unterliegen ebenfalls der genannten Creative Commons Lizenz, sofern sich aus der Abbildungslegende nichts anderes ergibt. Sofern das betreffende Material nicht unter der genannten Creative Commons Lizenz steht und die betreffende Handlung nicht nach gesetzlichen Vorschriften erlaubt ist, ist für die oben aufgeführten Weiterverwendungen des Materials die Einwilligung des jeweiligen Rechteinhabers einzuholen.

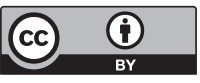

